Check for updates

Cite this: RSC Adv., 2018, 8, 20234

Received 8th May 2018

DOI: $10.1039 / c 8 r a 03948 h$

rsc.li/rsc-advances
Accepted 13th May 2018

\section{Influence of the nuclear charge distribution and electron correlation effects on magnetic shieldings and spin-rotation tensors of linear molecules $\uparrow$}

\author{
I. Agustín Aucar, (D) * Carlos A. Giménez and Gustavo A. Aucar (iD)
}

\begin{abstract}
The nuclear charge distribution effects (NChDE) on two response properties, the NMR magnetic shielding $(\boldsymbol{\sigma})$ and the nuclear spin-rotation (SR) constants (M), are analyzed. We do it employing point-like and Gaussian-like models for describing the nuclear charge density of three linear molecules: $\mathrm{HBr}, \mathrm{HI}$ and HAt. According to our results, both properties are sensitive to the NChDE. We show that the NChDE are almost completely relativistic, i.e., they nearly vanish in the non-relativistic limit of both properties. We calculated the NChDE on $\boldsymbol{\sigma}$ and $M$, and analyzed the differences between them in terms of a relativistic relation between these two properties. Using that relation we found that the electronic core mechanisms are the main ones for the NChDE on the shielding of nuclei of both, molecules and free atoms. The NChDE are smaller on SR constants than on shieldings. Nevertheless, within the relativistic polarization propagator formalism at the RPA level of approach they are very important for SR constants of nuclei in heavy-atom-containing compounds. Astatine in HAt has the largest influence: $M_{\text {At }}=$ $-9.95 \mathrm{kHz}$ for a point-like model and $-50.10 \mathrm{kHz}$ for a Gaussian-like model. Correlation effects must be included and we do it using different DFT schemes. The PBEO functional gives results that are closest to experiments for $\mathrm{Br}$ and I, though the LDA gives the closest for hydrogen. The value of the SR constant of At is reduced among $350 \mathrm{kHz}$ and $500 \mathrm{kHz}$ from its RPA value, when different and usual functionals are applied. Given that the NChDE on $M$ and $\boldsymbol{\sigma}$ are mostly relativistic in their origin, these effects are also dependent on electron correlation. They have also a nonvanishing dependence with the Gaunt electron-electron interactions.
\end{abstract}

\section{Introduction}

Accurate representation of the electronic structure of atoms and molecules, together with its properties, requires to consider the nuclear model as a finite one. This is specially the case for studies performed within a relativistic framework. ${ }^{1}$ Besides, phenomenologically modeled distributions of the nuclear charge and the dipolar nuclear magnetization are, till present, enough to get precise theoretical results. At the moment one does not need to resort to nuclear structure calculations.

Different types of both distributions can broadly be divided in two: point-like or finite-like. For the last one the first and usual option for electronic structure calculations is the spherically symmetric Gaussian-like nuclear model, due to its easy computational implementation. The electronic wave function is

Instituto de Modelado e Innovación Tecnológica, CONICET, Departamento de Física Facultad de Ciencias Exactas y Naturales, UNNE, Av. Libertad 5460, W3404AAS, Corrientes, Argentina. E-mail: agustin.aucar@conicet.gov.ar

$\dagger$ Electronic supplementary information (ESI) available: It contains tables of total isotropic shieldings and nuclear spin-rotation constants for $\mathrm{HBr}$ and $\mathrm{HI}$ molecules. It also contains a more detailed description of the information given about HAt. See DOI: 10.1039/c8ra03948h usually expanded in terms of Gaussian-type functions, and therefore the electron-nucleus attraction integrals appearing in the molecular calculations are easier to evaluate by employing the same efficient primitive integral routines used to evaluate the electron-electron repulsion integrals. In addition, this model depends on only one parameter, and the calculations are only sensitive to the rms charge radius. ${ }^{1-3}$

In 1993 Shabaev proposed few finite nuclear size corrections to the energy levels of multicharged ions ${ }^{4}$ and then, Visscher and Dyall were among the first to include the nuclear charge distribution effects, NChDE, in the calculation of the atomic ground state electronic energy., ${ }^{2,3}$ They considered different nuclear models and found that significant differences only appears when employing point-like or finite-like nuclear models. Their calculations gave close results for any of the following three different finite-size nuclear models: the homogeneously charged sphere, the two-parameter Fermi distribution and the Gaussian charge distribution. Few years later the NChDE were also included in electronic structure calculations of atoms and molecules by Andrae. ${ }^{5}$ He proposed an scheme to detect different values of physical properties when they are calculated using different finite nuclear models in standard quantum chemical electronic structure codes; specially in the 
total energy shifts and also in the energy differences in hydrogen-like atoms. One of the main conclusions of all these studies was that, to calculate the energy spectra, it is only relevant the switching from a point-like nuclear model to any of the finite-like nuclear charge distributions.

During the 90's, only the influence of the finite nuclear size models on calculations of wave functions and energies were carefully studied. More recently, considering that the hyperfine operator acts very close to the nucleus, the NChDE have become of particular interest for heavy-element-containing systems. Several works have been devoted to include such nuclear models in the expression of hyperfine operators. We can mention the work of Fukuda et al. about NMR chemical shifts at Douglas-Kroll-Hess (DKH) level of approach employing a finitenuclear model, ${ }^{6}$ and the work of Hamaya et al. whom included the NChDE on NMR shieldings $(\boldsymbol{\sigma})$ of halogen halides. ${ }^{7}$ In line with this, E. Malkin and co-workers have studied the effect of a finite nuclear model on the hyperfine structure at DKH-2/DFT level of theory ${ }^{8}$ and later at Dirac-Kohn-Sham (DKS) level of theory. ${ }^{9}$

Concerning the NChDE on NMR spectroscopic parameters, Autschbach has employed a Gaussian-type nuclear charge distribution model to calculate its influence on $J$-couplings. ${ }^{\mathbf{1 0 , 1 1}}$ In this case the relativistic zeroth-order regular approach (ZORA), combined with both non-hybrid and hybrid density functionals were applied, and both, spin-free and twocomponent spin-orbit terms were calculated. It was shown that the NChDE on the hyperfine integrals are quite pronounced, and therefore, they noticeably alter $J$-coupling constants when heavy atoms are involved. Few years later some of us found that relativistic effects largely enhance the NChDE on those parameters and also that $J$-couplings are more sensitive than shieldings in both, relativistic and non-relativistic (NR) regimes. The highest effect, which is larger than $10 \%$ of variation was found for $J$-couplings among lead and iodine in $\mathrm{PbIH}_{3}{ }^{12}$

Kita and Tachikawa ${ }^{13}$ have also found that the nuclear size effects on $\boldsymbol{\sigma}$, and also on the molecular magnetic susceptibility, may be important for heavy-element-containing systems. Furthermore, Arcisauskaite et al. used a Gaussian-type model to include the NChDE on shielding constants in $\mathrm{HgX}_{2}(\mathrm{X}=\mathrm{Cl}, \mathrm{Br}$, $\left.\mathrm{I}, \mathrm{CH}_{3}\right){ }^{\mathbf{1 4}}$ They found that the NChDE are larger when fourcomponent calculations are performed, as compared with ZORA results.

In the case of the relativistic spin-rotation constants, its first calculations (presented at the 10th REHE International Conference in 2012) were performed with a finite nuclear charge distribution model. It is also worth to highlight the fact that a Gaussian-type nuclear charge distribution model was employed in the first two published articles with calculations of $\boldsymbol{M}$, performed at the relativistic four-component Dirac-KohnSham ${ }^{15}$ and Dirac-Hartree-Fock levels of theory. ${ }^{16}$ In any case, until now no detailed studies of NChDE on this property were made.

Given that the relativistic nuclear spin-rotation (SR) tensor $(\boldsymbol{M})^{17-19}$ is closely related to the NMR shielding tensor, or turning this statement around, the relativistic extension of Flygare's relationship ${ }^{20,21}$ shows that the NMR shielding tensor is related with the SR tensor, the shielding constant of free atoms and a new term (see below) named $\boldsymbol{v}^{\mathrm{S}}$, one may expect that the NChDE should be important for $\boldsymbol{M}$. On the other hand, given that electron correlation and relativistic effects are not independent each other for the NMR spectroscopic parameters, ${ }^{22}$ there may be a relationship among electron correlation and NChDE if these last effects are enhanced by relativistic effects, as seems to be the case. The main goals of this work are related with the answers to these two inquiries. We shall analyze the NChDE on the SR constants by the first time, and consider also its relativistic relationship with the shielding constants in some details, to shed some light on the electronic origin of the NChDE in both properties. Besides the previously mentioned aims of this work, another one is the analysis of the electron correlation as related with the total NChDE. Furthermore, we analyzed the electron correlation effects treated at the relativistic DFT level of theory, on both properties and also on its NChDE. We shall also show how important could be the consideration of the two-electron (SS|SS) integrals at the DiracFock level on the NChDE, and also the electron-electron Gaunt interactions.

This article has the following structure: in Section 2 we briefly introduce a description of the nuclear charge distribution models we use. We also present in that Section a summary of the backgrounds of the relation between $\boldsymbol{M}$ and $\boldsymbol{\sigma}$ within a relativistic framework. In Section 3 we describe in some detail how our calculations were performed and then, an analysis of the NChDE on SR and $\sigma$ is presented in Section 4, where a systematic study of the origin of these effects is exposed. It includes the analysis of the Gaunt interactions. The main conclusions are given in Section 5.

\section{Theory and models}

\subsection{Nuclear charge distribution models}

The use of finite nuclear models in electronic structure calculations modifies the electrostatic Coulomb electron-nucleus potential. Its most important consequence is associated with the change from a point-like nucleus (with a singularity at the nuclear position), to some spherically symmetric one. ${ }^{1}$

There are some nuclear models available in the bibliography. In this work, we focus on one-dimensional spherically symmetric models for the nuclear charge density distributions, because the tridimensional models are not yet implemented in the computational codes commonly used to calculate the properties of our interest. There are four basic types of onedimensional nuclear charge distributions (NChD): the pointlike nuclear model and three finite-size distributions. Between the latter, we can recognize the uniform, the Gaussian-type and the Fermi-type distributions. In our case we focus our attention to only two of those models: the point-type one and the Gaussian-type one.

It is known that the differences between potential energy functions obtained from the three different finite-size nuclear distributions are always moderate. ${ }^{5}$ 
The nuclear charge density distribution of both, point-type and spherically symmetric Gaussian-type of a nucleus of atomic number $Z\left(\rho_{\mathrm{P}}(r)\right.$ and $\rho_{\mathrm{G}}(r)$, respectively), can be written as

$$
\rho_{\mathrm{P}}(r)=Z \delta(r) ; \rho_{\mathrm{G}}(r)=\rho_{\mathrm{G}, 0} \mathrm{e}^{-\lambda r^{2}},
$$

where $\rho_{\mathrm{G}, 0}$ is fixed through the normalization condition

$$
\rho_{\mathrm{G}, 0}=Z\left(\frac{\lambda}{\pi}\right)^{\frac{3}{2}} ; \lambda=\frac{3}{2\left\langle R^{2}\right\rangle} .
$$

Atomic units were used in the last expressions and will be adopted throughout all this work.

The nuclear charge density distributions $\rho_{\mathrm{P}}(r)$ and $\rho_{\mathrm{G}}(r)$ give rise to spherically symmetric potentials, given as

$$
\begin{gathered}
V_{\mathrm{P}}(r)=-\frac{Z}{r}, \\
V_{\mathrm{G}}(r)=-\frac{Z}{r} \operatorname{erf}(\sqrt{\lambda} r),
\end{gathered}
$$

where $\operatorname{erf}(x)$ denotes the error function, which is the probability that a measurement error will be between $-x$ and $x$, and is given by

$$
\operatorname{erf}(x)=\frac{2}{\sqrt{\pi}} \int_{0}^{x} \mathrm{e}^{-t^{2}} \mathrm{~d} t
$$

The main parameter used to describe the Gaussian-type distribution is the rms nuclear radius $\sqrt{\left\langle R^{2}\right\rangle}$, which can be approximately related to the cubic root of the mass number $A$ of the given nucleus via the empirical relation ${ }^{23}$ $\sqrt{\left\langle R^{2}\right\rangle}=\left(0.836 A^{1 / 3}+0.570\right) \mathrm{fm}$.

\subsection{NMR shieldings and spin-rotation constants}

We now turn to the relationship among the shielding and SR constants. Since the first decades of the NMR spectroscopy, a close relationship between the NMR shielding tensor of a nucleus $\mathrm{Y}, \boldsymbol{\sigma}_{\mathrm{Y}}$, and its nuclear spin-rotation tensor $\boldsymbol{M}_{\mathrm{Y}}$ was broadly used to determine absolute scales of shieldings. It was first proposed by Ramsey ${ }^{24}$ and then improved by Flygare, ${ }^{25,26}$ being very useful until our days. Such a relationship was derived within a NR framework, and is expressed (in atomic units) as

$$
\begin{aligned}
\boldsymbol{\sigma}_{\mathrm{Y}} & =\boldsymbol{\sigma}_{\mathrm{Y}}^{\mathrm{NR}-\mathrm{para}}+\boldsymbol{\sigma}_{\mathrm{Y}}^{\mathrm{NR}-\mathrm{dia}} \\
& \approx \frac{m_{\mathrm{p}}}{g_{\mathrm{Y}}} \boldsymbol{M}_{\mathrm{Y}}^{\mathrm{NR}} \otimes \boldsymbol{I}+\boldsymbol{\sigma}_{\mathrm{Y}}^{\text {atom,NR }} .
\end{aligned}
$$

This relation was recently found to be not any longer valid within the relativistic framework. In eqn (6), $m_{\mathrm{p}}$ is the proton mass, $g_{\mathrm{Y}}$ is the nuclear $g$-value of nucleus $\mathrm{Y}$, and $\boldsymbol{I}$ is the molecular moment tensor of inertia in the equilibrium geometry, with respect to its center of mass. Besides Flygare have shown that eqn (6) is more accurate for the isotropic values than for each individual tensor element. ${ }^{26-28}$
The Ramsey-Flygare relation of eqn (6) was recently generalized to the relativistic framework. ${ }^{20,21}$ The new model can be used to obtain absolute shieldings.

The spin-rotation tensor of a nucleus $\mathrm{Y}$ in a molecule in its equilibrium position (for which the electric field at each nucleus is zero) can be expressed as a sum of two terms $\left(\boldsymbol{M}_{\mathbf{Y}}=\boldsymbol{M}_{\mathrm{Y}}^{\text {nuc }}+\right.$ $\left.\boldsymbol{M}_{\mathrm{Y}}^{\text {elec }}\right)$. One of them depends only on nuclear variables $\left(\boldsymbol{M}_{\mathrm{Y}}^{\text {nuc }}\right)$ whereas the second one includes the electronic dependence $\left(\boldsymbol{M}_{\mathrm{Y}}^{\text {elec }}\right) \cdot{ }^{17,26}$

Working within the four-component polarization propagator, the tensor $\boldsymbol{M}_{\mathrm{Y}}^{\text {elec }}$ can be splitted into two terms that arises by considering separately the electronic excitations from occupied positive-energy orbitals to unoccupied positive-energy orbitals (e-e contributions) and to negative-energy ones ( $\mathrm{p}-\mathrm{p}$ contributions). ${ }^{29,30}$ The tensor $\boldsymbol{M}_{\mathrm{Y}}^{\text {elec }}$ can be expressed as

$$
\boldsymbol{M}_{\mathrm{Y}}^{\text {elec }}=\frac{g_{\mathrm{Y}}}{2 m_{\mathrm{p}} c^{2}}\left\langle\left\langle\left(\frac{\boldsymbol{r}-\boldsymbol{r}_{\mathrm{Y}}}{\left|\boldsymbol{r}-\boldsymbol{r}_{\mathrm{Y}}\right|^{3}} \times c \boldsymbol{\alpha}\right) ; \boldsymbol{J}_{\mathrm{e}}\right\rangle\right\rangle \otimes \boldsymbol{I}^{-1}
$$

where $\ll$; stand for the relativistic polarization propagator, $\boldsymbol{\alpha}$ represent the Dirac matrices, and the relativistic electronic total angular momentum operator, $\boldsymbol{J}_{e}=\left(\boldsymbol{r}-\boldsymbol{r}_{\mathrm{CM}}\right) \times \boldsymbol{p}+\frac{1}{2} \boldsymbol{\Sigma}$, is the sum of the corresponding orbital and spin angular momenta. The orbital angular momentum is considered with respect to the molecular center of mass, and $\boldsymbol{\Sigma}$ is the fourcomponent extension of the Pauli matrices. It must be highlighted that eqn (7) does not include the effects of the Breit electron-nucleus interactions. They are not treated at all in the present work. The very small influence of these effects was analyzed in a previous work. ${ }^{31}$

On the other hand, the tensor $\boldsymbol{\sigma}_{\mathrm{Y}}$ is written in the polarization propagator formalism as

$$
\boldsymbol{\sigma}_{\mathrm{Y}}=\frac{1}{2 c^{2}}\left\langle\left\langle\left(\frac{\boldsymbol{r}-\boldsymbol{r}_{\mathrm{Y}}}{\left|\boldsymbol{r}-\boldsymbol{r}_{\mathrm{Y}}\right|^{3}} \times c \boldsymbol{\alpha}\right) ;\left(\boldsymbol{r}-\boldsymbol{r}_{\mathrm{G}}\right) \times c \boldsymbol{\alpha}\right\rangle\right\rangle
$$

where $\boldsymbol{r}_{\mathrm{G}}$ stands for the position of the (arbitrary) gauge origin of the magnetic potential.

In order to extend the NR Ramsey-Flygare relation to the relativistic domain we started from the recognition of common electronic mechanisms in both $\boldsymbol{\sigma}_{\mathrm{Y}}$ and $\boldsymbol{M}_{\mathbf{Y}}$ tensors, first applying the linear response within the elimination of the small components (LRESC) model, and then generalizing it to the four-component case. ${ }^{17,20,21,32}$ Few other research groups also published recently other developments about the relativistic extension of Ramsey-Flygare's relationship. ${ }^{15,19}$

Assuming that the LRESC model is reliable in reproducing the leading order (in a $\frac{1}{c^{2}}$ expansion) relativistic effects of $S R$ and shielding constants for both, its (e-e) and (p-p) contributions, we proposed few models that link both properties within a relativistic framework. ${ }^{20,21}$ The most precise of them, the model $\mathrm{M}-\mathrm{V}$, relates $\boldsymbol{\sigma}_{\mathrm{Y}}$ and $\boldsymbol{M}_{\mathrm{Y}}$ as follows:

$$
\boldsymbol{\sigma}_{\mathrm{Y}}^{\mathrm{M}-\mathrm{V}}=\frac{m_{\mathrm{p}}}{g_{\mathrm{Y}}} \boldsymbol{M}_{\mathrm{Y}} \otimes \boldsymbol{I}+\boldsymbol{\sigma}_{\mathrm{Y}}^{\text {atom }}+\frac{1}{2 c}\left(\boldsymbol{\nu}_{\mathrm{Y}}^{\mathrm{S}}-\boldsymbol{\nu}_{\mathrm{Y}}^{\text {atom }, \mathrm{S}}\right),
$$


where $\boldsymbol{\sigma}_{\mathrm{Y}}^{\text {atom }}$ is the shielding tensor of the nucleus $\mathrm{Y}$ for the free atom, and $\nu_{\mathrm{Y}}^{\mathrm{S}}$ is defined as: ${ }^{21}$

$$
\nu_{\mathrm{Y}}^{\mathrm{S}}=\frac{1}{c}\left\langle\left\langle\left(\frac{\boldsymbol{r}-\boldsymbol{r}_{\mathrm{Y}}}{\left|\boldsymbol{r}-\boldsymbol{r}_{\mathrm{Y}}\right|^{3}} \times c \boldsymbol{\alpha}\right) ; \boldsymbol{S}_{\mathrm{e}}\right\rangle\right\rangle,
$$

where $S_{\mathrm{e}}=\frac{1}{2} \boldsymbol{\Sigma}$.

In the special case of linear molecules, the tensor $\boldsymbol{\sigma}_{\mathrm{Y}}$ can be written as a diagonal matrix, whose elements are $\sigma_{\perp, \mathrm{Y}}$ (simply degenerate) and $\sigma_{\|, \mathrm{Y}}$. This last term is the shielding of the nucleus $\mathrm{Y}$ when the applied magnetic field is aligned parallel to the molecular bond axis, whereas $\sigma_{\perp, \mathrm{Y}}$ is the shielding experienced by the nucleus when the magnetic field is aligned perpendicular to the bond axis.

The model $\mathrm{M}-\mathrm{V}$, applied to a linear molecule gives

$$
\sigma_{\perp, \mathrm{Y}}^{\mathrm{M}-\mathrm{V}(\mathrm{e}-\mathrm{e})}=\frac{m_{\mathrm{p}} I}{g_{\mathrm{Y}}} M_{\perp, Y}^{\mathrm{elec}(\mathrm{e}-\mathrm{e})}+\sigma_{\mathrm{Y}}^{\text {atom }(\mathrm{e}-\mathrm{e})}+\frac{1}{2 c}\left(\nu_{\perp, \mathrm{Y}}^{\mathrm{S}(\mathrm{e}-\mathrm{e})}-\nu_{\mathrm{Y}}^{\mathrm{atom}, \mathrm{S}(\mathrm{e}-\mathrm{e})}\right)
$$

$$
\begin{aligned}
\sigma_{\perp, \mathrm{Y}}^{\mathrm{M}-\mathrm{V}(\mathrm{p}-\mathrm{p})}= & \frac{m_{\mathrm{p}} I}{g_{\mathrm{Y}}}\left(M_{\perp, \mathrm{Y}}^{\mathrm{nuc}}+M_{\perp, \mathrm{Y}}^{\text {elec }(\mathrm{p}-\mathrm{p})}\right)+\sigma_{\mathrm{Y}}^{\text {atom }(\mathrm{p}-\mathrm{p})} \\
& +\frac{1}{2 c}\left(\nu_{\perp, \mathrm{Y}}^{\mathrm{S}(\mathrm{p}-\mathrm{p})}-\nu_{\mathrm{Y}}^{\text {atom }, \mathrm{S}(\mathrm{p}-\mathrm{p})}\right),
\end{aligned}
$$

and

$$
\begin{gathered}
\sigma_{\|, \mathrm{Y}}^{\mathrm{M}-\mathrm{V}(\mathrm{e}-\mathrm{e})}=\sigma_{\mathrm{Y}}^{\text {atom }(\mathrm{e}-\mathrm{e})}+\frac{1}{2 c}\left(\nu_{\|, \mathrm{Y}}^{\mathrm{S}(\mathrm{e}-\mathrm{e})}-\nu_{\mathrm{Y}}^{\text {atom }, \mathrm{S}(\mathrm{e}-\mathrm{e})}\right) \\
\sigma_{\|, \mathrm{Y}}^{\mathrm{M}-\mathrm{V}(\mathrm{p}-\mathrm{p})}=\sigma_{\mathrm{Y}}^{\text {atom }(\mathrm{p}-\mathrm{p})}+\frac{1}{2 c}\left(\nu_{\|, \mathrm{Y}}^{\mathrm{S}(\mathrm{p}-\mathrm{p})}-\nu_{\mathrm{Y}}^{\text {atom }, \mathrm{S}(\mathrm{p}-\mathrm{p})}\right) .
\end{gathered}
$$

Therefore, the isotropic shielding constant for linear molecules $\left(\sigma_{\mathrm{Y}}^{\text {iso }}=\frac{2 \sigma_{\perp, \mathrm{Y}}+\sigma_{\|, \mathrm{Y}}}{3}\right)$ can be expressed, according to the model $\mathrm{M}-\mathrm{V}$, as

$$
\boldsymbol{\sigma}_{\mathrm{Y}}^{\mathrm{M}-\mathrm{V} \text {,iso }}=\frac{2}{3} \frac{m_{\mathrm{p}} I}{g_{\mathrm{Y}}} M_{\perp, \mathrm{Y}}+\sigma_{\mathrm{Y}}^{\text {atom }}+\frac{1}{2 c}\left(\nu_{\mathrm{Y}}^{\mathrm{S} \text {,iso }}-\nu_{\mathrm{Y}}^{\text {atom }, \mathrm{S}}\right),
$$

where $M_{\perp, \mathrm{Y}}$ is the perpendicular component of the spinrotation tensor (simply called "nuclear spin-rotation constant”, $M$, for linear molecules).

\section{Computational details}

Four-component relativistic calculations of shielding and SR tensor elements for three linear molecules were performed using a locally modified developer version of the DIRAC16 (ref. 33) program package.

The previous nomenclature that pointed out any nucleus as $Y$ shall now be enlarged to introduce a distinction between the heavy nucleus and the hydrogen. The $\mathrm{HX}\left(\mathrm{X}={ }^{79} \mathrm{Br},{ }^{127} \mathrm{I},{ }^{210} \mathrm{At}\right)$ molecules are our model systems. They were displayed along the $z$ axis. For symmetry reasons, as we deal with linear molecules, only diagonal components of shielding and SR tensors are no null. In the case of the $\boldsymbol{M}$ tensor, in both relativistic and NR domains only its perpendicular components are non-zero and equal each other. These tensor elements are known as the spin-rotation constant $M$. On the other side, within the relativistic domain the shielding tensor has nonzero parallel $\left(\sigma_{\|}\right.$ $\left.=\sigma_{z z}\right)$ and perpendicular $\left(\sigma_{\perp}=\sigma_{x x}=\sigma_{y y}\right)$ elements. However, in the NR case $\sigma_{\|}^{\mathrm{NR}}=\sigma_{z z}^{\mathrm{NR}}=0$.

Unless otherwise stated, four-component calculations are based on the Dirac-Coulomb Hamiltonian, employing the default Hamiltonian of the DIRAC code. It uses an energy correction to avoid the explicit calculation of (SS|SS) integrals, i.e., two-electron integrals containing only small component basis functions. ${ }^{34}$ In addition, in some appropriately indicated calculations the (SS|SS) integrals were also included in order to analyze the importance of this contribution. Furthermore, the most precise calculation of this work are based on the DiracCoulomb-Gaunt Hamiltonian (where, due to implementation reasons, the Breit electron-electron interaction is replaced by the Gaunt interaction, i.e. neglecting the retardation terms), including explicitly the calculation of (SS|SS) integrals. We assume that the Gaunt interaction provides a useful approximation to the Breit interaction; it is considered to be an order of magnitude larger than the retardation term..$^{35,36}$

Most of the response calculations were performed within the Dirac-Hartree-Fock-Coulomb relativistic polarization propagator approach at the random phase level of approach (RPA). Nevertheless, some of the RPA calculations were performed taking into account the Gaunt electron-electron interaction included in the unperturbed Hamiltonian. They are explicitly indicated as such. In addition, to analyze the influence of electron correlation effects within the Dirac-Hartree-FockCoulomb (-Gaunt) framework, some calculations employing the pure zeroth-order approximation (PZOA) were performed. ${ }^{37}$

Non-relativistic values of $\boldsymbol{\sigma}$ and $\boldsymbol{M}$ (reported from here as $\boldsymbol{\sigma}^{\mathrm{NR}-\text { para }}, \boldsymbol{\sigma}^{\mathrm{NR}-\mathrm{dia}}$ and $\left.\boldsymbol{M}^{\mathrm{NR}-\mathrm{elec}}\right)$ were obtained taking a speed of light of $c=30 c_{0}$. The value of the speed of light in vacuum used throughout all four-component calculations was $c_{0}=$ 137.0359998 a.u.

The gauge origin of the external magnetic potential was placed at the molecular center of mass in the NMR shielding calculations. With this choice, a direct comparison with the SR results can be safely made. Furthermore, in order to calculate shieldings of nuclei in closed shell free atoms using the DIRAC code (this feature is not implemented for open shell systems), an electron was added to the halogen free atoms.

The following values of nuclear $\mathrm{g}$-factors were taken from ref. 38 for the calculations of SR constants: 5.585694 for ${ }^{1} \mathrm{H}$, 1.404267 for ${ }^{79} \mathrm{Br}$ and 1.125309 for ${ }^{127} \mathrm{I}$. As experimental data does not exist for ${ }^{210} \mathrm{At}$, a $g$-factor of 1.0 was chosen for this nucleus.

Experimental bond distances were extracted from ref. 39 for $\mathrm{HBr}$ and HI. For HAt, an optimized distance was used and calculated at the RPA level of approach. They are: $1.4145 \AA$ (HBr), $1.6090 \AA$ (HI) and $1.7117 \AA$ (HAt).

In all calculations, the NR Dunning's augmented correlationconsistent aug-cc-pCV5Z basis set was used for the hydrogen atom. ${ }^{40}$ For Br, I and At the Dyall's relativistic acv4z basis sets (dyall.acv4z) were employed. ${ }^{41}$ In all cases, the uncontracted Gaussian basis sets were used with the common gauge-origin (CGO) approach. The small component basis sets for 
Table 1 SR constants conversion factors. The third column values are expressed in atomic units $\left(\frac{\mathrm{ppm}}{\text { Hartree }}\right)$, whereas those of the fourth column are given in $\frac{\mathrm{ppm}}{\mathrm{kHz}}$

\begin{tabular}{|c|c|c|c|c|}
\hline \multirow[b]{2}{*}{$\mathrm{mol}$} & \multirow[b]{2}{*}{$Y$} & $m_{\mathrm{p}} I(\mathrm{~mol})$ & [ $\mathrm{ppm}$ & $m_{\mathrm{p}} I(\mathrm{~mol})[\mathrm{ppm}]$ \\
\hline & & $g_{\mathrm{Y}}$ & {$[\overline{\text { Hartree }}$} & $\overline{\mathrm{kHz}}$ \\
\hline \multirow[t]{2}{*}{$\mathrm{HBr}$} & $\mathrm{Br}$ & 16.94603 & $\times 10^{12}$ & 2.57550783 \\
\hline & $\mathrm{H}$ & 4.26058 & $\times 10^{12}$ & 0.64753560 \\
\hline \multirow[t]{2}{*}{ HI } & I & 27.49115 & $\times 10^{12}$ & 4.17818744 \\
\hline & $\mathrm{H}$ & 5.53938 & $\times 10^{12}$ & 0.84189143 \\
\hline \multirow[t]{2}{*}{ HAt } & At & 35.12509 & $\times 10^{12}$ & 5.33841677 \\
\hline & $\mathrm{H}$ & 6.28840 & $\times 10^{12}$ & 0.95573026 \\
\hline
\end{tabular}

relativistic calculations were generated by applying the unrestricted kinetic balance prescription (UKB).

Point and finite nuclear models-modeled by Gaussian charge distributions-(PNM and GNM, respectively) were employed in all calculations, as implemented in the DIRAC code.

In order to study correlation effects, we performed PZOA, RPA and Kohn-Sham-DFT calculations employing the DIRAC code. The DFT calculations are based on the four-component Dirac-Coulomb Hamiltonian, and have been done using a variety of NR exchange-correlation functionals in several categories: (i) The local density approximation (LDA) functional, ${ }^{42,43}$ (ii) the generalized gradient approximation (GGAs) functionals: PBE, ${ }^{44} \mathrm{KT} 2{ }^{45} \mathrm{KT} 3{ }^{46}$ and $\mathrm{BP} 86,{ }^{47,48}$ and (iii) the hybrid functional PBE0. ${ }^{49}$ Furthermore, calculations based on the Dirac-Coulomb-Gaunt Hamiltonian were performed employing the PBE0 functional, scaling the Gaunt integrals (with the GAUNTSCALE keyword of the DIRAC program) with the same factor as for Hartree-Fock exchange. It means that this hybrid functional include fractional Hartree-Fock Gaunt interaction.

For the property calculations at DFT level of theory, experimental internuclear distances were used for $\mathrm{HBr}$ and $\mathrm{HI}$. The optimized bond distances of the HAt molecule were again obtained from each of the above mentioned functionals and basis sets. Their values are (in $\AA$ ) 1.7393 (LDA), 1.7237 (KT2), 1.7209 (KT3), 1.7473 (PBE), 1.7272 (PBE0) and 1.7486 (BP86). Doing this we are as much coherent as possible with the scheme of calculations adopted.

In addition, Table 1 gives the conversion factors $\frac{m_{\mathrm{p}} I}{g_{\mathrm{Y}}}$ used to transform the SR constants from atomic units (Hartree) to ppm (3rd column of the table), to be used in the application of eqn (6) and (9). In the fourth column the conversion factors of SR constants from $\mathrm{kHz}$ to $\mathrm{ppm}$ are given. They are obtained by taking into account the relation between Hartrees and $\mathrm{kHz}$ $\left(1\right.$ Hartree $\left.=6.579683920711 \times 10^{12} \mathrm{kHz}\right) .{ }^{50}$

\section{Results and discussion}

In this Section, the influence of the NChDE on the (e-e) and (p-p) contributions to $\boldsymbol{\sigma}_{\mathrm{Y}}$ and $\boldsymbol{M}_{\mathrm{Y}}$ are analyzed separately in order to understand the origin of these effects. In line with this, the NChDE are studied on the shielding of both, nuclei in free atoms and nuclei in molecules. In addition, their NR limit are also analyzed. Furthermore, the model $\mathrm{M}-\mathrm{V}$ is employed to study the underlying physics of the NChDE in $\sigma_{\mathrm{Y}}$. Finally, an analysis of how much the electronic correlation affects the NChDE is presented.

\subsection{Four-component RPA calculations}

In order to study the NChDE on $\sigma_{\mathrm{Y}}$ and $M_{\mathrm{Y}}$, the relativistic RPA values of $\sigma_{\perp, \mathrm{Y}}^{(\mathrm{e}-\mathrm{e})}, \frac{m_{\mathrm{P}} I}{g_{\mathrm{Y}}} \boldsymbol{M}_{\perp, \mathrm{Y}}^{(\mathrm{e}-\mathrm{e})}$, and the NR limit of both, $\sigma_{\perp, \mathrm{Y}}^{\mathrm{NR}-\mathrm{para}}$, calculated with PNM and GNM are shown in Table 2.

In addition, in Table 3 we show the values of $\sigma_{\perp, \mathrm{Y}}^{(\mathrm{p}-\mathrm{p})}, \frac{m_{\mathrm{p}} I}{g_{\mathrm{Y}}} M_{\perp, \mathrm{Y}}^{(\mathrm{p}-\mathrm{p})}$ and $\sigma_{\perp, \mathrm{Y}}^{\mathrm{NR}-\text { dia }}$ also calculated employing both, GNM and PNM.

The SR constants are multiplied by the factor $\frac{m_{\mathrm{p}} I}{g_{\mathrm{Y}}}$, which is the quotient between $\sigma_{\perp, \mathrm{Y}}^{\mathrm{NR}-\mathrm{Y} a r a}$ and $M_{\perp, \mathrm{Y}}^{\mathrm{NR} \text {-elec }}$ (the NR limits of $\sigma_{\perp, \mathrm{Y}}^{(\mathrm{e}-\mathrm{e})}$ and $M_{\perp, \mathrm{Y}}^{(\mathrm{e}-\mathrm{e})}$, respectively) as proposed by the Flygare's NR relation: ${ }^{25}$

$$
\sigma_{\perp, \mathrm{Y}}^{\mathrm{NR}-\mathrm{para}}=\frac{m_{\mathrm{p}} I}{g_{\mathrm{Y}}} M_{\perp, \mathrm{Y}}^{\mathrm{NR}-\text { elec }}
$$

Given that $M_{\perp, Y}^{\text {elec }}$ can be expressed as the sum of two terms $\left(M_{\perp, \mathrm{Y}}^{\text {elec }}=M_{\perp, \mathrm{Y}}^{\mathrm{L}}+M_{\perp, \mathrm{Y}}^{\mathrm{S}}\right.$; see in eqn (7) that $\left.\boldsymbol{J}_{\mathrm{e}}=\boldsymbol{L}_{\mathrm{e}}+\boldsymbol{S}_{\mathrm{e}}\right)$, its values are splitted up into $M_{\perp, \mathrm{Y}}^{\mathrm{L}}$ and $M_{\perp, \mathrm{Y}}^{\mathrm{s}}$. In addition, the NChDE values for each property are displayed in Tables 2 and 3.

The NChDE on $\boldsymbol{\sigma}_{\mathrm{Y}}$ and $\boldsymbol{M}_{\mathrm{Y}}$ are obtained as the differences between the values of each property employing Gaussian-type and point-type nuclear charge distribution models. Throughout this work, unless otherwise stated, the symbol $\Delta$ will be exclusively employed to refer to these differences (for example, $\Delta \boldsymbol{\sigma}_{\mathrm{Y}}=\boldsymbol{\sigma}_{\mathrm{Y}}^{\mathrm{GNM}}-\boldsymbol{\sigma}_{\mathrm{Y}}^{\mathrm{PNM}}$ ).

One important finding is that in all cases $\Delta M_{\perp, \mathrm{Y}}^{\mathrm{L}}$ and $\Delta M_{\perp, \mathrm{Y}}^{\mathrm{S}}$ have opposite signs, as shown in the last two columns of Tables 2 and 3. For the heavy nucleus $\mathrm{X}, \Delta M_{\perp, \mathrm{X}}^{\mathrm{S}(\mathrm{e}) \mathrm{e}}$ grows faster than $\Delta M_{\perp, \mathrm{X}}^{\mathrm{L}(\mathrm{e}) \mathrm{e})}$ as the atomic number of the $\mathrm{X}$ atom increases $(\mathrm{X}=$ $\mathrm{Br}, \mathrm{I}, \mathrm{At})$. The opposite behavior is found for the hydrogen nucleus.

The relative value of the NChDE in both properties needs a special mention. Its importance with respect to the relativistic effects on $M_{\perp, \mathrm{Y}}^{(\mathrm{e}-\mathrm{e})}$ and $\sigma_{\perp, \mathrm{Y}}^{(\mathrm{e}-\mathrm{e})} \quad\left(i . e . \frac{\Delta M_{\perp, \mathrm{Y}}^{(\mathrm{e}-\mathrm{e})}}{M_{\perp, \mathrm{Y}}^{(\mathrm{e}-\mathrm{e})}-M_{\perp, \mathrm{Y}}^{\mathrm{NR}-\mathrm{elec}}}\right.$ and $\left.\frac{\Delta \sigma_{\perp, \mathrm{Y}}^{(\mathrm{e}-\mathrm{e})}}{\sigma_{\perp, \mathrm{Y}}^{(\mathrm{e}-\mathrm{e})}-\sigma_{\perp, \mathrm{Y}}^{\mathrm{NR}-\text { para }}}\right)$ increases from $-2.5 \%$ (SR) and $-0.4 \%$ (shielding) for $\mathrm{Br}$, up to $-9.0 \%$ (SR) and $-6.0 \%$ (shielding) for At (see Table 2). This means that the NChDE grows faster than the relativistic effects, specially for shieldings.

It is also worth to highlight the fact that $\Delta \sigma_{\perp, Y}^{(\mathrm{e}-\mathrm{Y})}$ represents $1.2 \%$ of $\sigma_{\perp, \mathrm{Y}}^{(\mathrm{e}-\mathrm{e})}$ for $\mathrm{Y}=\mathrm{Br}$, and it grows up to $7.6 \%$ for $\mathrm{Y}=\mathrm{At}$. On the other hand, $\Delta M_{\perp, \mathrm{Y}}^{(\mathrm{e}-\mathrm{e})}$ represents $0.03 \%$ of $M_{\perp, \mathrm{Y}}^{(\mathrm{e}-\mathrm{e})}$ when $\mathrm{Y}=\mathrm{Br}$ and increases up to $77.5 \%$ for $\mathrm{Y}=\mathrm{At}$ !

According to our results, we can state that the choice of the nuclear model used on relativistic calculations of shieldings 
Table 2 Calculated values of $\boldsymbol{\sigma}_{\perp}^{\mathrm{NR}-\text { para }}, \boldsymbol{M}_{\perp}^{(\mathrm{e}-\mathrm{e})}$ and $\boldsymbol{\sigma}_{\perp}^{(\mathrm{e}-\mathrm{e})}$ at the RPA level of approach using both, PNM and GNM. All values are given in ppm

$$
\underline{\frac{m_{\mathrm{p}} \boldsymbol{I}}{g_{\mathrm{Y}}} \boldsymbol{M}_{\perp, \mathrm{Y}}^{(\mathrm{e}-\mathrm{e})}}
$$

NChDE

\begin{tabular}{|c|c|c|c|c|c|c|c|c|c|}
\hline \multirow[b]{2}{*}{ Molec } & \multirow[b]{2}{*}{$\mathrm{Y}$} & & \multirow[b]{2}{*}{$\boldsymbol{\sigma}_{\perp, \mathrm{Y}}^{\mathrm{NR}-\mathrm{para}}$} & \multirow[b]{2}{*}{$\mathrm{L}$} & \multirow[b]{2}{*}{$\mathrm{S}$} & \multirow[b]{2}{*}{$\boldsymbol{\sigma}_{\perp, \mathrm{Y}}^{(\mathrm{e}-\mathrm{e})}$} & \multirow[b]{2}{*}{$\Delta \sigma_{\perp, \mathrm{Y}}^{(\mathrm{e}-\mathrm{e})}$} & \multicolumn{2}{|c|}{$\frac{m_{\mathrm{p}} \boldsymbol{I}}{g_{\mathrm{Y}}} \Delta \boldsymbol{M}_{\perp, \mathrm{Y}}^{(\mathrm{e}-\mathrm{e})}$} \\
\hline & & & & & & & & $\mathrm{L}$ & $\mathrm{S}$ \\
\hline \multirow[t]{4}{*}{$\mathrm{HBr}$} & $\mathrm{Br}$ & PNM & -742.0222 & -933.3812 & 199.2331 & -187.3019 & -2.3174 & 0.6091 & -0.8015 \\
\hline & & GNM & -742.0164 & -932.7721 & 198.4316 & -189.6193 & & & \\
\hline & $\mathrm{H}$ & PNM & 16.6034 & 25.6238 & -0.2920 & 25.0586 & -0.0002 & -0.0003 & 0.0000 \\
\hline & & GNM & 16.6034 & 25.6235 & -0.2920 & 25.0584 & & & \\
\hline \multirow[t]{4}{*}{$\mathrm{HI}$} & I & PNM & -1454.4561 & -2114.7400 & 754.8454 & 650.5588 & -22.9512 & 5.6493 & -8.0874 \\
\hline & & GNM & -1454.4296 & -2109.0907 & 746.7580 & 627.6076 & & & \\
\hline & $\mathrm{H}$ & PNM & 17.8542 & 49.0594 & -2.0569 & 44.9705 & -0.0032 & -0.0037 & 0.0002 \\
\hline & & GNM & 17.8542 & 49.0557 & -2.0567 & 44.9673 & & & \\
\hline \multirow[t]{4}{*}{ HAt } & At & PNM & -2661.1988 & -4445.6835 & 4383.2938 & 11293.7041 & -794.5878 & 24.8291 & -239.2336 \\
\hline & & GNM & -2661.0624 & -4420.8544 & 4144.0602 & 10499.1163 & & & \\
\hline & $\mathrm{H}$ & PNM & 18.4044 & 147.3452 & -22.0295 & 103.3245 & -0.1183 & -0.1989 & 0.0404 \\
\hline & & GNM & 18.4044 & 147.1463 & -21.9891 & 103.2062 & & & \\
\hline
\end{tabular}

Table 3 Calculated values of $\boldsymbol{\sigma}_{\perp}^{\mathrm{NR}-\mathrm{dia}}, \boldsymbol{M}_{\perp}^{(\mathrm{p}-\mathrm{p})}$ and $\boldsymbol{\sigma}_{\perp}^{(\mathrm{p}-\mathrm{p})}$ at the RPA level of approach using both, PNM and GNM. All values are given in ppm

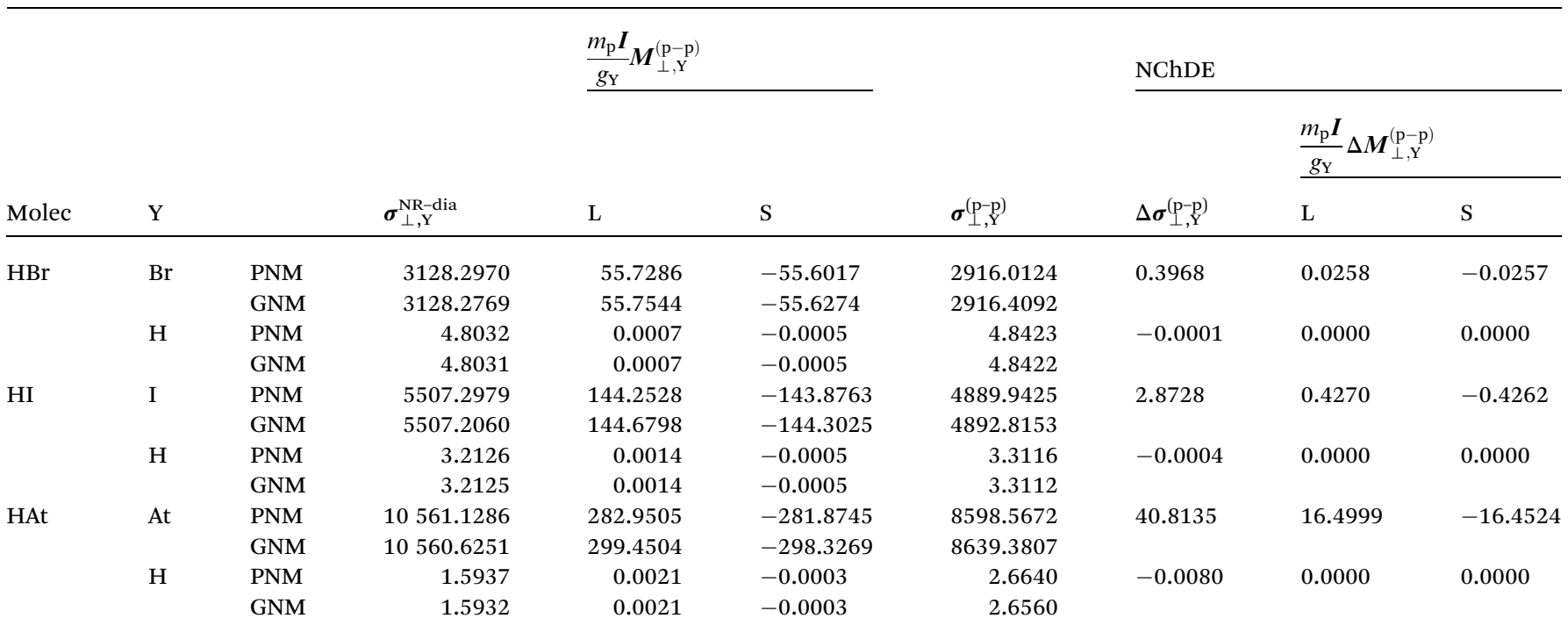

and SR constants is increasingly more important as heavier nuclei are involved. It can still be crucial for calculations of SR constants of nuclei belonging to the 6 th row of the periodic table.

As observed in Table 3, there are two important points to be highlighted for $\Delta \sigma_{\perp, \mathrm{Y}}^{(\mathrm{p}-\mathrm{p})}$ and $\Delta M_{\perp, \mathrm{Y}}^{(\mathrm{p}-\mathrm{p})}$. The first one is that $\Delta M_{\perp, \mathrm{Y}}^{(\mathrm{p}-\mathrm{p})}$ is close to zero for all nuclei. This behavior can be understood by considering the LRESC model, from which the NR limit and the leading order in a $\frac{1}{c^{2}}$ expansion of $M_{\perp, \mathrm{Y}}^{(\mathrm{p}-\mathrm{p})}$ are found to be zero. ${ }^{17}$ The nucleus of At has the largest value, where $\frac{m_{\mathrm{p}} I}{g_{\mathrm{At}}} \Delta M_{\perp, \mathrm{At}}^{(\mathrm{p}-\mathrm{p})}=0.0475 \mathrm{ppm}$ and $\frac{m_{\mathrm{p}} I}{g_{\mathrm{At}}} M_{\perp, \mathrm{At}}^{(\mathrm{p}-\mathrm{p})}=1.1235 \mathrm{ppm}$.
The second point is that $\Delta \sigma_{\perp, \mathrm{Y}}^{(\mathrm{p}-\mathrm{p})}$ is less than $0.5 \%$ of $\sigma_{\perp, \mathrm{Y}}^{(\mathrm{p}-\mathrm{p})}$ for all Y nuclei. Therefore, the NChDE have an influence that is greater on the $(e-e)$ than on the $(p-p)$ contributions for both properties.

\subsection{The NR limit}

By applying the relativistic polarization propagator formalism the NR limit of $\Delta \boldsymbol{M}_{\mathrm{Y}}$ and $\Delta \boldsymbol{\sigma}_{\mathrm{Y}}$ can be straightforwardly obtained. According to this theory the NR limit is reached when the speed of light is scaled to infinity. This is the reason why a set of calculations of the tensor elements of $\boldsymbol{M}_{\mathrm{Y}}$ and $\boldsymbol{\sigma}_{\mathrm{Y}}$ was performed considering the two nuclear models, and systematically increasing the velocity of light; we employed 32 values of $c$ between $c_{0}$ and $10 c_{0}$. An smooth convergence of $\Delta \boldsymbol{M}_{\mathrm{Y}}$ and $\Delta \boldsymbol{\sigma}_{\mathrm{Y}}$ to values that are close to zero was found in all cases. 

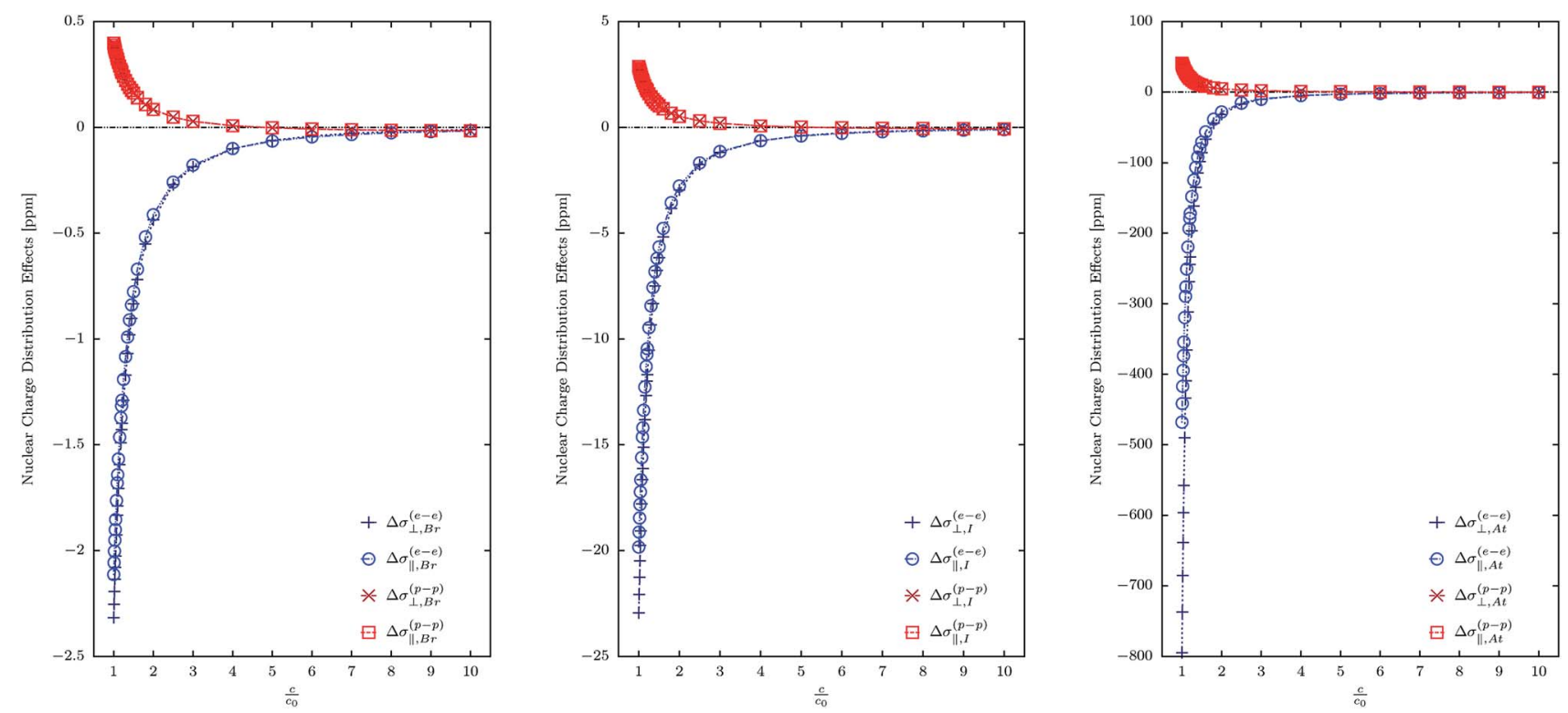

Fig. 1 NChDE on the perpendicular and parallel components of the shielding constants of nuclei $X$ in $H X(X=B r, l, A t)$ as a function of the scaling factor of the speed of light, $\frac{c}{c_{0}}$. The $(e-e)$ and $(p-p)$ contributions are shown separately. Calculations were performed at the RPA level of approach.

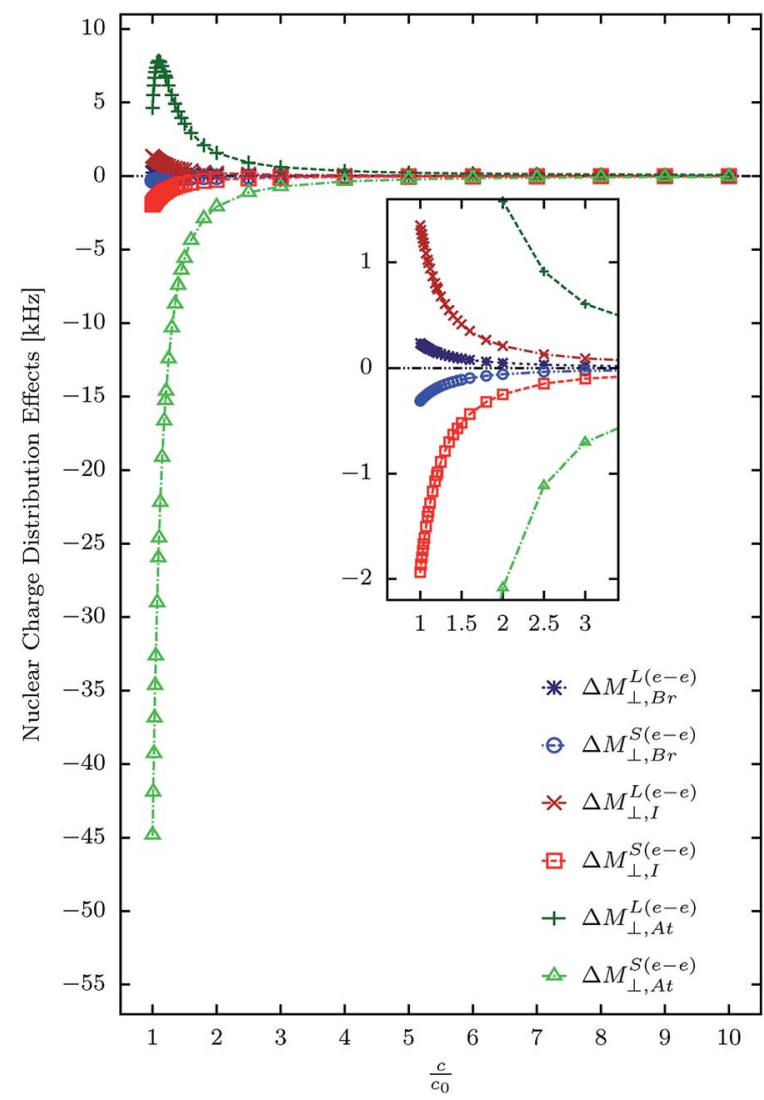

Fig. $2 \mathrm{NChDE}$ on $M_{\perp, \mathrm{X}}^{(\mathrm{e}-\mathrm{e})}$ in the heavy nuclei $\mathrm{X}$ of the $\mathrm{HX}(\mathrm{X}=\mathrm{Br}, \mathrm{l}, \mathrm{At})$ molecules, as a function of the scaling factor of the speed of light, $\frac{c}{c_{0}}$. The $L$ and $S$ contributions to the SR constants are displayed separately in order to show their opposite behavior. Calculations were performed at the RPA level of approach.
In Fig. 1 and 2 one can observe the dependence of the (e-e) and (p-p) contributions to $\Delta \boldsymbol{\sigma}_{\mathrm{X}}$ and $\Delta \boldsymbol{M}_{\mathrm{X}}$ with the velocity of light, respectively. It is shown that all of them become vanishingly small as $c$ scales to infinity.

In Fig. 1 we observe that there is a higher rate of change of the (e-e) contributions as compared with the (p-p) contributions. This indicates a higher sensitivity of $\Delta \boldsymbol{\sigma}_{\mathrm{X}}^{(\mathrm{e}-\mathrm{e})}$ to the relativistic effects compared with $\Delta \sigma_{\mathrm{X}}^{(\mathrm{p}-\mathrm{p})}$. Furthermore, $\Delta \sigma_{\perp, \mathrm{X}}^{(\mathrm{e}-\mathrm{e})}$ is more sensitive to the relativistic effects than $\Delta \sigma_{\|, \mathrm{X}}^{(\mathrm{e}-\mathrm{e})}$; this is not the behavior of the $(\mathrm{p}-\mathrm{p})$ contributions.

On the other hand, in Fig. 2 we show how $\Delta M_{\perp, \mathrm{X}}^{\mathrm{L}(\mathrm{e}-\mathrm{e})}$ and $\Delta M^{\mathrm{S}(\mathrm{e}-}$ e) ${ }_{\perp, \mathrm{x}}$ go to zero as $c$ scales to infinity. A higher slope of $\Delta M_{\perp, \mathrm{X}}^{\mathrm{S}(\mathrm{e}-\mathrm{e})}$ than that of $\Delta M_{\perp, \mathrm{X}}^{\mathrm{L}(\mathrm{e}-\mathrm{e})}$ is also observed. It means that $\Delta M_{\perp, \mathrm{X}}^{\mathrm{S}(\mathrm{e}-\mathrm{e})}$ is most sensitive than $\Delta M_{\perp, \mathrm{X}}^{\mathrm{L}(\mathrm{e}-\mathrm{e})}$ to the relativistic effects.

It is worth to mention that for At, $\frac{m_{\mathrm{p}} I}{g_{\mathrm{At}}} \Delta M_{\perp, \mathrm{At}}^{\mathrm{S}(\mathrm{e}-\mathrm{e})}$ changes from $-239.23 \mathrm{ppm}$ to $-5.95 \mathrm{ppm}$ (about $+233 \mathrm{ppm}$ of variation) when the speed of light is scaled from $c_{0}$ to $2.5 c_{0}$. For the same values of $c, \frac{m_{\mathrm{p}} I}{g_{\mathrm{At}}} M_{\perp, \mathrm{At}}^{\mathrm{L}(\mathrm{e}-\mathrm{e})}$ changes only $-20 \mathrm{ppm}$ (from 24.83 ppm to 4.88 ppm).

We should emphasize here that the values of $\sigma_{\|, \mathrm{Y}}^{(\mathrm{e}-\mathrm{e})}, M_{\perp, \mathrm{Y}}^{\mathrm{S}(\mathrm{e}-\mathrm{e})}, M_{\perp, \mathrm{Y}}^{\mathrm{L}(\mathrm{p}-\mathrm{p})}$ and $M_{\perp, \mathrm{Y}}^{\mathrm{S}(\mathrm{p}-\mathrm{p})}$ are exactly zero in the NR limit, and therefore, their NChDE vanish in such a limit. Comparing eqn (7) with eqn (10) it can be seen that

$$
\frac{1}{2 c} \nu_{\perp, \mathrm{Y}}^{\mathrm{S}}=\frac{m_{\mathrm{p}} I}{g_{\mathrm{Y}}} M_{\perp, \mathrm{Y}}^{\mathrm{S}} .
$$

Then, it indicates that $\nu_{\perp, \mathrm{Y}}^{\mathrm{S}(\mathrm{e}-\mathrm{e})}$ and $\nu_{\perp, \mathrm{Y}}^{\mathrm{S}(\mathrm{p}-\mathrm{p})}$ and their NChDE are also exactly zero in the NR limit. Following the same argument, the NChDE on $\sigma_{\mathrm{Y}}^{\text {atom(e-e) }}$ and $\frac{1}{2 c} \nu_{\mathrm{Y}}^{\text {atom,s }}$ are zero in such limit. 
Therefore, we can state that $\Delta \sigma_{\|, \mathrm{Y}}^{(\mathrm{e}-\mathrm{e})}, \Delta M_{\perp, \mathrm{Y}}^{\mathrm{S}(\mathrm{e}-\mathrm{e})}, \Delta M_{\perp, \mathrm{Y}}^{(\mathrm{p}-\mathrm{p})}, \Delta \sigma_{\mathrm{Y}}^{\text {atom }(\mathrm{e}-\mathrm{e})}, \Delta \nu_{\perp, \mathrm{Y}}^{\mathrm{S}(\mathrm{e}-\mathrm{e})}, \Delta \nu_{\perp, \mathrm{Y}}^{\mathrm{S}(\mathrm{p}-\mathrm{p})}$ and $\frac{1}{2 c} \Delta \nu_{\mathrm{Y}}^{\text {atom,S }}$ can have values that are different from zero only within the relativistic framework. All this means that only $\Delta \sigma_{\perp, \mathrm{Y}}^{(\mathrm{e}-\mathrm{e})}, \Delta M_{\perp, \mathrm{Y}}^{\mathrm{L}(\mathrm{e}-\mathrm{e})}, \Delta \sigma_{\mathrm{Y}}^{(\mathrm{p}-\mathrm{p})}$ and $\Delta \sigma_{\mathrm{Y}}^{\text {atom(p-p) }}$ may have values that are very small in the NR limit, but not exactly zero.

Still, according to eqn (11)-(14), the following NR limits are fulfilled:

$$
\lim _{c \rightarrow \infty} \Delta \sigma_{\perp, \mathrm{Y}}^{(\mathrm{e}-\mathrm{e})}=\lim _{c \rightarrow \infty} \frac{m_{\mathrm{p}} I}{g_{\mathrm{Y}}} \Delta M_{\perp, \mathrm{Y}}^{\mathrm{L}(\mathrm{e}-\mathrm{e})}=\Delta \sigma_{\perp, \mathrm{Y}}^{\mathrm{NR}-\text { para }}
$$

and

$$
\lim _{c \rightarrow \infty} \Delta \sigma_{\mathrm{Y}}^{(\mathrm{p}-\mathrm{p})} \simeq \lim _{c \rightarrow \infty} \Delta \sigma_{\mathrm{Y}}^{\text {atom }(\mathrm{p}-\mathrm{p})}=\Delta \sigma_{\mathrm{Y}}^{\text {atom, }, \mathrm{NR}}
$$

As shown in Fig. 1 and 2, they are vanishingly small.

\subsection{Relation between the $\sigma_{\mathrm{Y}}$ and $M_{\mathrm{Y}}$ tensors}

In this Section we want to shed some light on the influence of the NChDE on each of the four terms of the $\mathrm{M}-\mathrm{V}$ model given in eqn (9). We shall see that the first two terms of eqn (9) contain almost the whole of the NChDE of $\boldsymbol{\sigma}$ in a relativistic framework

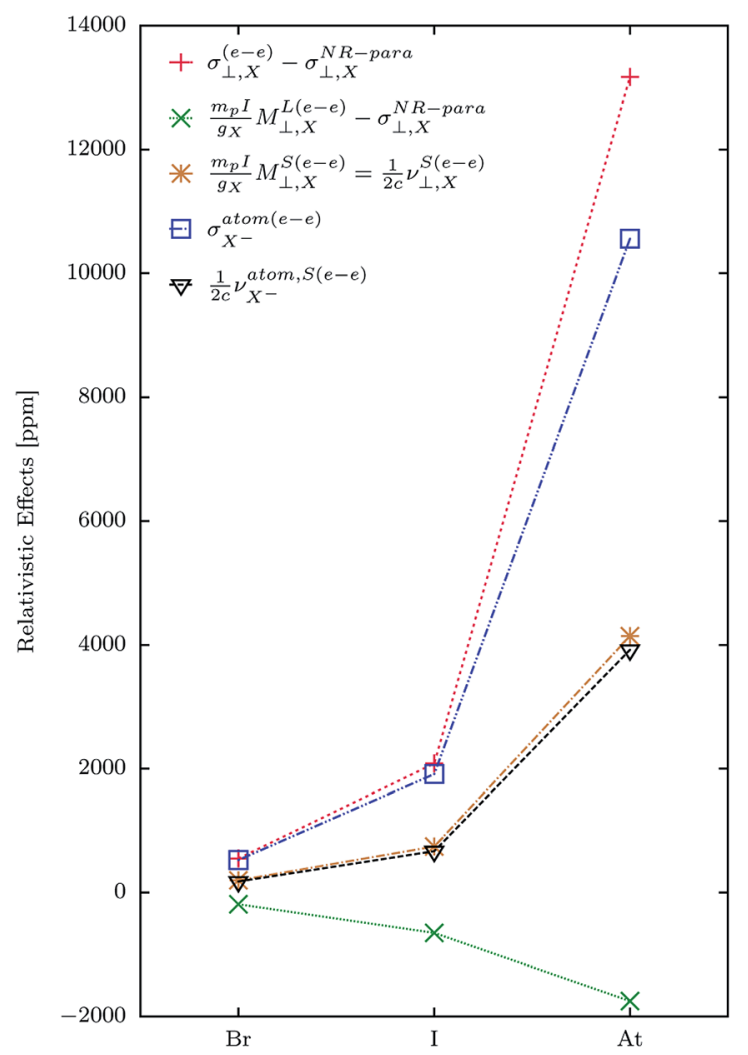

Fig. 3 Relativistic effects on perpendicular (e-e) contributions to shielding and SR constants ( $L$ and S components are shown separately) of the heavy nuclei $X$ in $H X(X=B r, I, A t)$ molecules. Also $\sigma^{\text {atom }}$ and $v^{\text {atom, }}$ are displayed. Calculations were performed at the RPA level of approach using Gaussian nuclear model. All values are given in ppm. because each one of the last two are close each other. Therefore we will focus our analysis on these terms.

In Fig. 3 the relativistic effects (i.e., the differences between four-component and NR calculations using a GNM) on $\sigma_{\perp, \mathrm{X}}^{(\mathrm{e}-\mathrm{e})}, M_{\perp, \mathrm{X}}^{\mathrm{L}(\mathrm{e}-\mathrm{e})}$ and $M_{\perp, \mathrm{X}}^{\mathrm{S}(\mathrm{e}-\mathrm{e})}$ for the heavy nuclei X are shown. The values of $\sigma_{\mathrm{X}}^{\text {atom(e-e) }}$, that has only relativistic contributions, are also given.

It can easily be seen in Fig. 3 that the main relativistic contribution to $\sigma_{\perp, \mathrm{X}}^{(\mathrm{e}-\mathrm{e})}$ comes from the shielding of the free atom $\mathrm{X}$, followed far away by that of the SR constant (which is given by $\left.\frac{m_{\mathrm{p}} I}{g_{\mathrm{X}}} M_{\perp, \mathrm{X}}^{\mathrm{L}(\mathrm{e}-\mathrm{e})}+\frac{m_{\mathrm{p}} I}{g_{\mathrm{X}}} M_{\perp, \mathrm{X}}^{\mathrm{S}(\mathrm{e}-\mathrm{e})}-\sigma_{\perp, \mathrm{X}}^{\mathrm{NR}-\mathrm{para}}\right)$. Besides, the relativistic effects on $M_{\perp, \mathrm{X}}^{\mathrm{L}}$ and $M_{\perp, \mathrm{X}}^{\mathrm{S}}$ have opposite signs, which reduces the total relativistic effect of this property.

The value of the relativistic effect on $\frac{m_{\mathrm{p}} I}{g_{\mathrm{At}}} M_{\perp, \mathrm{At}}^{(\mathrm{e}-\mathrm{e})}$ is 2384.27 ppm, whereas for $\sigma_{\mathrm{At}^{-}}^{\text {atom }(\mathrm{e}-\mathrm{e})}$ it is $10557.13 \mathrm{ppm}$ (remember that it has no NR counterpart). The addition of both values gives $12941.40 \mathrm{ppm}$, which is close to the total relativistic effect of $\sigma_{\perp, \mathrm{At}}^{(\mathrm{e}-\mathrm{e})}: 13160.18 \mathrm{ppm}$. According to the $\mathrm{M}-\mathrm{V}$ model (see eqn (11)) the remaining difference between those values arises from $\frac{1}{2 c}\left(\nu_{\perp, \mathrm{At}}^{\mathrm{S}}-\nu_{\mathrm{At}^{-}}^{\text {ato } \mathrm{S}}\right)$, which is equal to $219.49 \mathrm{ppm}$. It must be emphasized that $\nu_{\perp, \mathrm{Y}}^{\mathrm{S}}$ and $\nu_{\mathrm{Y}}^{\text {atom,S }}$ are zero in the NR limit.

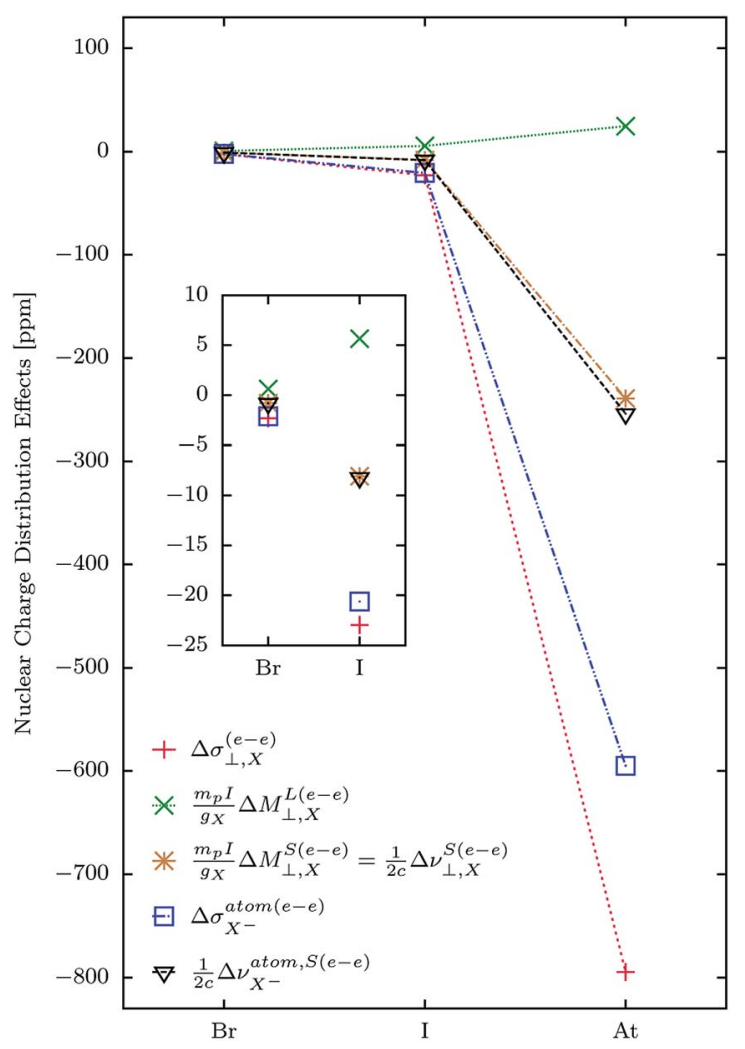

Fig. 4 NChDE on perpendicular (e-e) contributions to shielding and SR constants ( $L$ and $S$ components are shown separately) of the heavy nuclei in $\mathrm{HX}\left(\mathrm{X}=\mathrm{Br}\right.$, I, At) molecules. Also NChDE on $\boldsymbol{\sigma}^{\text {atom }}$ and $v^{\text {atom, }}$ are displayed. Calculations were performed at the RPA level of approach. All values are given in ppm. 
Table 4 Values of $(e-e)$ and $(p-p)$ contributions to $\boldsymbol{\sigma}_{Y^{-}}^{\text {atom }}$ and $\frac{1}{2 c} \boldsymbol{\nu}_{Y^{-}}^{\text {atom, }}$ at the RPA level of approach using PNM and GNM. All values are in ppm

\begin{tabular}{llrrrr}
\hline \multicolumn{1}{l}{$\mathrm{Y}$} & & $\boldsymbol{\sigma}_{\mathrm{Y}^{-}}^{\text {atom }(\mathrm{e}-\mathrm{e})}$ & $\boldsymbol{\sigma}_{\mathrm{Y}^{-}}^{\text {atom }(\mathrm{p}-\mathrm{p})}$ & $\frac{1}{2 c} \boldsymbol{\nu}_{\mathrm{Y}^{-}}^{\text {atom, } \mathrm{S}(\mathrm{e}-\mathrm{e})}$ & $\frac{1}{2 c} \boldsymbol{\nu}_{\mathrm{Y}^{-}}^{\text {atom, } \mathrm{S}(\mathrm{p}-\mathrm{p})}$ \\
\hline $\mathrm{Br}$ & $\mathrm{PNM}$ & 528.2667 & 2913.4249 & 180.4978 & -55.5860 \\
& GNM & 526.1324 & 2913.8216 & 179.6876 & -55.6119 \\
$\mathrm{I}$ & $\mathrm{PNM}$ & 1934.1137 & 4887.3336 & 678.2519 & -143.7614 \\
& GNM & 1913.4712 & 4890.2057 & 670.0392 & -144.1902 \\
At & PNM & 11152.2334 & 8595.1847 & 4176.7886 & -279.9905 \\
& GNM & 10557.1342 & 8635.9570 & 3922.8385 & -296.5936
\end{tabular}

Another interesting finding is the fact that $\frac{1}{2 c} \nu_{\perp, \mathrm{X}}^{\mathrm{S}(\mathrm{e}-\mathrm{e})}$ and $\frac{1}{2 c} \nu_{\mathrm{X}}^{\text {atom, } \mathrm{S}(\mathrm{e}-\mathrm{e})}$ are close each other (for $\mathrm{X}=\mathrm{Br}, \mathrm{I}, \mathrm{At}$ ) and so, given that they contribute with different sign, its total contribution becomes small.

The NChDE on SR and shielding tensors have an almost complete relativistic origin, which explain the behavior of different contributions to the NChDE shown in Fig. 4. The main contribution to $\Delta \sigma_{\perp, \mathrm{X}}^{(\mathrm{e}-\mathrm{e})}$ in the $\mathrm{HX}$ systems comes from the NChDE on the free-atom shielding, followed by $\frac{m_{\mathrm{p}} I}{g_{\mathrm{X}}} \Delta M_{\perp, \mathrm{X}}^{\mathrm{S}(\mathrm{e}-\mathrm{e})}\left(=\frac{1}{2 c} \Delta \nu_{\perp, \mathrm{X}}^{\mathrm{S}(\mathrm{e}-\mathrm{e})}\right)$. In addition, $\Delta M_{\perp, \mathrm{X}}^{\mathrm{L}(\mathrm{e}-\mathrm{e})}$ is almost zero in all cases.

According to eqn (11), $\frac{m_{\mathrm{p}} I}{g_{\mathrm{X}}} M_{\perp, \mathrm{X}}^{\mathrm{S}(\mathrm{e}-\mathrm{e})}$ must be included two times in $\sigma_{\perp, \mathrm{X}}^{\mathrm{M}-\mathrm{V}}$. It can be seen in Fig. 4 that its NChDE is almost equal to $\frac{1}{2 c} \Delta \nu_{\mathrm{X}}^{\text {atom }, \mathrm{S}(\mathrm{e}-\mathrm{e})}$. Given that $\nu_{\perp, \mathrm{X}}^{\mathrm{S}}$ and $\nu_{\mathrm{X}}^{\text {atom, } \mathrm{S}}$ have opposite signs in eqn (11), the sum of their NChDE is almost zero.

Finally, in Table 4 we display the values of the calculations of $\sigma_{\mathrm{X}^{-}}^{\text {atom }}$ and $\frac{1}{2 c} \nu_{\mathrm{X}^{-}}^{\text {atom,S }}$, used to obtain $\sigma_{\perp, \mathrm{Y}}^{\mathrm{M}-\mathrm{V}}$ and $\sigma_{\|, \mathrm{Y}}^{\mathrm{M}-\mathrm{V}}$ according to eqn (9)-(15).

\subsection{Isotropic SR and shielding constants}

We turn now to the analysis of how important are the NChDE in measurable quantities, such as the total SR constants and the chemical shifts.

In Table 5 it is observed that the NChDE on $\sigma_{\mathrm{Y}}^{\text {iso }}$ of all heavy elements are more important for (e-e) than for ( $\mathrm{p}-\mathrm{p})$ contributions, although they have opposite signs and so partially cancel each other. As the nucleus becomes heavier, also the difference increases in such a way that for At $\Delta \sigma_{\mathrm{At}}^{\text {iso(e-e) }} \cong-686 \mathrm{ppm}$, while $\Delta \sigma_{\mathrm{At}}^{\text {iso(p-p) }} \cong+41 \mathrm{ppm}$. It means that almost all the NChDE on $\sigma_{\mathrm{At}}^{\text {iso }}$ arises from its (e-e) contribution, which is known to be related to the (e-e) part of the SR constant.

For hydrogen in $\mathrm{HX}, \Delta \sigma_{\mathrm{Y}}^{\text {iso }}$ has also a greater (e-e) contribution than its (p-p) counterpart, but the sum of both represent only a small contribution to the total shielding. The NChDE is completely negligible for the shielding of hydrogen in this series of compounds. For $\mathrm{H}$ in HAt, $\Delta \sigma_{\mathrm{H}}^{\text {iso }}=$ $-0.06 \mathrm{ppm}$, and the total isotropic shielding is $\sigma_{\mathrm{H}}^{\text {iso }}=$ 74.90 ppm.

Table 5 Isotropic values of the shielding and SR constants at the RPA level of approach. Values between parenthesis correspond to calculations using London atomic orbitals. All values are in ppm

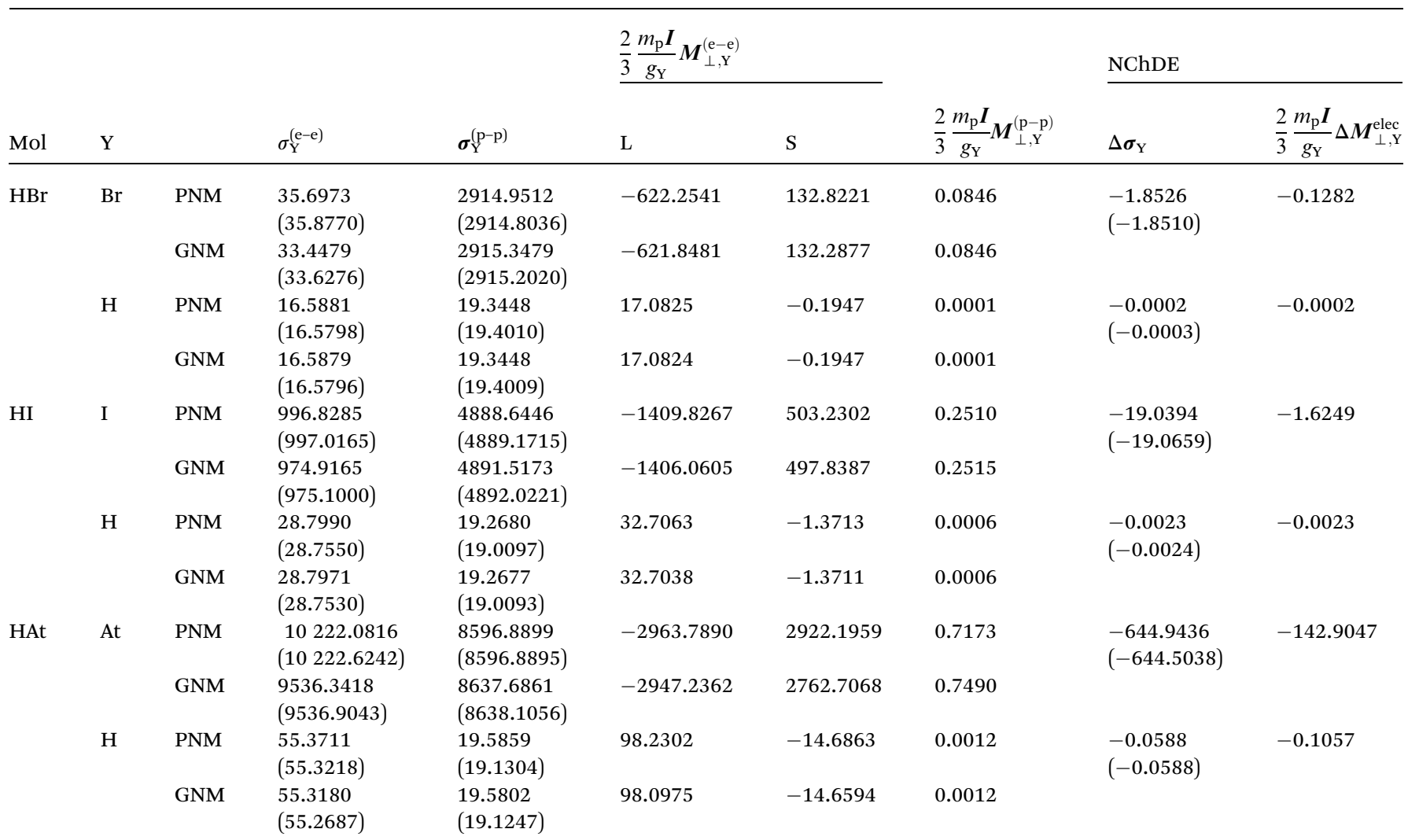




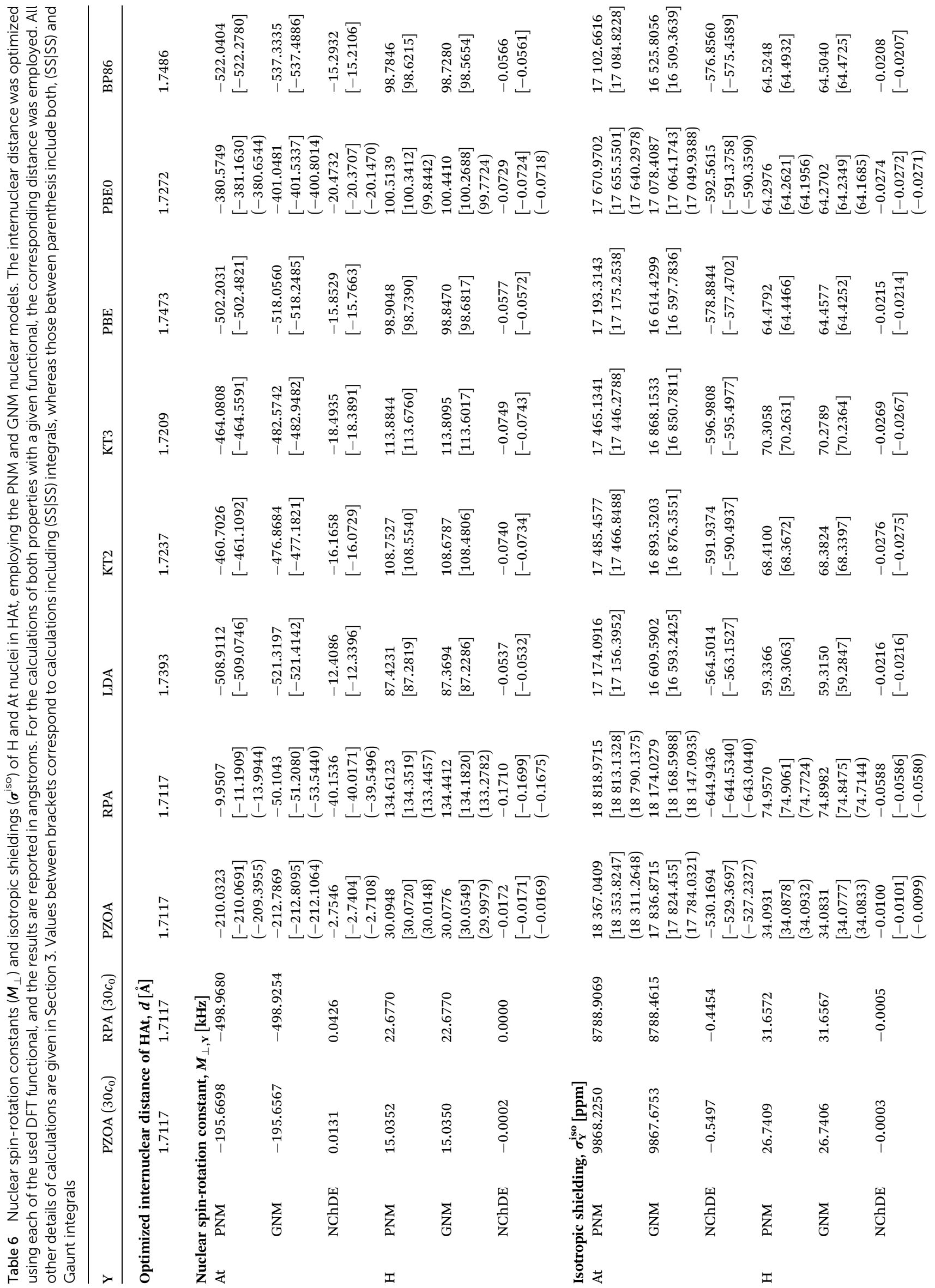


The analysis of the isotropic SR constant is unnecessary, as it would be completely analogous to that of the perpendicular contribution to the SR tensor performed above. This occurs because the parallel component of $\boldsymbol{M}_{\mathrm{Y}}$ is always exactly zero for linear molecules. Nevertheless, as its isotropic value is related to the isotropic shielding constant, some remarks must be done. The contributions $\Delta \boldsymbol{M}_{Y}^{S(e-e)}$ are more important than $\Delta \boldsymbol{M}_{Y}^{\mathrm{L}(\mathrm{e}-\mathrm{e})}$, as it was shown in Section 4.1. In the special case of At, the NChDE must be included in the 4-component RPA calculations. When a PNM is used, a value of $\frac{2}{3} \frac{m_{\mathrm{p}} I}{g_{\mathrm{At}}} M_{\perp, \mathrm{At}}^{\text {elec }}=-40.87 \mathrm{ppm}$ is obtained, whereas it becomes $-183.78 \mathrm{ppm}$ when a GNM is employed.

Although the NChDE has an important contribution to $\sigma_{\mathrm{At}}^{\text {iso }}$ in the HAt molecule $\left(\Delta \sigma_{\mathrm{At}}^{\text {iso }} \cong-645 \mathrm{ppm}\right.$, whereas $\sigma_{\mathrm{At}}^{\text {iso }} \cong$ $18174 \mathrm{ppm})$, the SR constant of this nucleus cannot be calculated with a PNM, at least at the RPA level of approach $\left(\Delta M_{\perp, \text { At }}\right.$ $\cong-40.15 \mathrm{kHz}$, whereas $M_{\perp, \mathrm{At}} \cong-50.10 \mathrm{kHz}$ ).

Finally it can be seen in Table 5 that $\frac{2}{3} \frac{m_{\mathrm{p}} I}{g_{\mathrm{At}}} \Delta M_{\perp, \mathrm{At}}=-142.90 \mathrm{ppm}$, whereas $\Delta \sigma_{\mathrm{At}}^{\text {iso }}=-644.94 \mathrm{ppm}$. If we consider the relation between isotropic SR and shielding constants of eqn (15), the difference of almost $-502 \mathrm{ppm}$ must

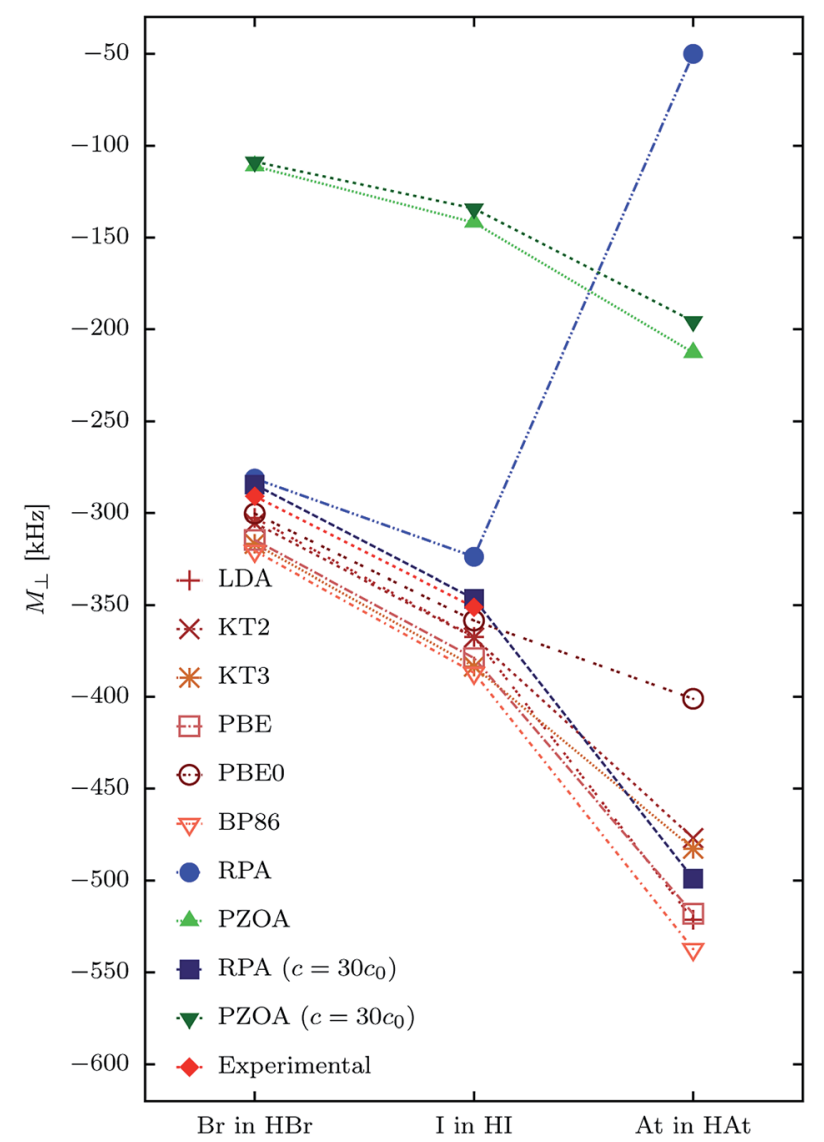

be originated in the NChDE on $\sigma_{\mathrm{At}^{-}}^{\text {atom }}, \frac{1}{2 c} \nu_{\mathrm{At}}^{\mathrm{S}-\text { iso }}$ and $\frac{1}{2 c} \nu_{\mathrm{At}^{-}}^{\text {atom, }}$. Those contributions are $-554.33 \mathrm{ppm},-218.30 \mathrm{ppm}$ and $-270.55 \mathrm{ppm}$, respectively (see Table 4). As expected, applying the model $\mathrm{M}-\mathrm{V}$ we found that $\Delta \sigma_{\mathrm{At}^{-}}^{\text {atom }}+\Delta \frac{1}{2 c} \nu_{\mathrm{At}}^{\mathrm{S}-\mathrm{iso}}-\Delta \frac{1}{2 c} \nu_{\mathrm{At}^{-}}^{\text {atom } \mathrm{S}}$ gives $-502 \mathrm{ppm}$.

\subsection{Electron correlation effects}

It is already known that the influence of electron correlation on both properties, $\boldsymbol{M}$ and $\boldsymbol{\sigma}$, is not vanishingly small. ${ }^{16,51-55}$ For this reason we want now to have an estimation of how large is the effect of electron correlation when it is included on top of the NChDE.

On the other hand, it was also proposed that one should consider a likely relation among the electron correlation and relativistic effects. ${ }^{51,56-60}$ This relation was recently shown to be not independent one to the other. ${ }^{22,54} \mathrm{So}$, it may be the case that the electron correlation and the NChDE are also dependent each other.

We have calculated both properties employing the set of the DFT functionals just mentioned. Their results are then

Fig. 5 Relativistic spin-rotation values of both nuclei in $\mathrm{HX}$ molecules (X $=\mathrm{Br}, \mathrm{I}, \mathrm{At}$ ). Results at PZOA and RPA levels of approach and also employing different DFT functionals are shown. In addition, PZOA and RPA results with $c=30 c_{0}$ are displayed in order to show the behavior of $M_{\perp}$ in the NR limit. All calculations were performed using the GNM, and the results are given in $\mathrm{kHz}$. Experimental values (in $\mathrm{kHz}$ ) are: $41.27(31)(\mathrm{H}$ in $\mathrm{HBr}){ }^{61}-290.83(8)(\mathrm{Br}$ in $\mathrm{HBr}){ }^{61}$ 49.22(22) $(\mathrm{H} \text { in } \mathrm{HI})^{62}$ and $-351.1(3)(\mathrm{I}$ in $\mathrm{HI}){ }^{62}$ 

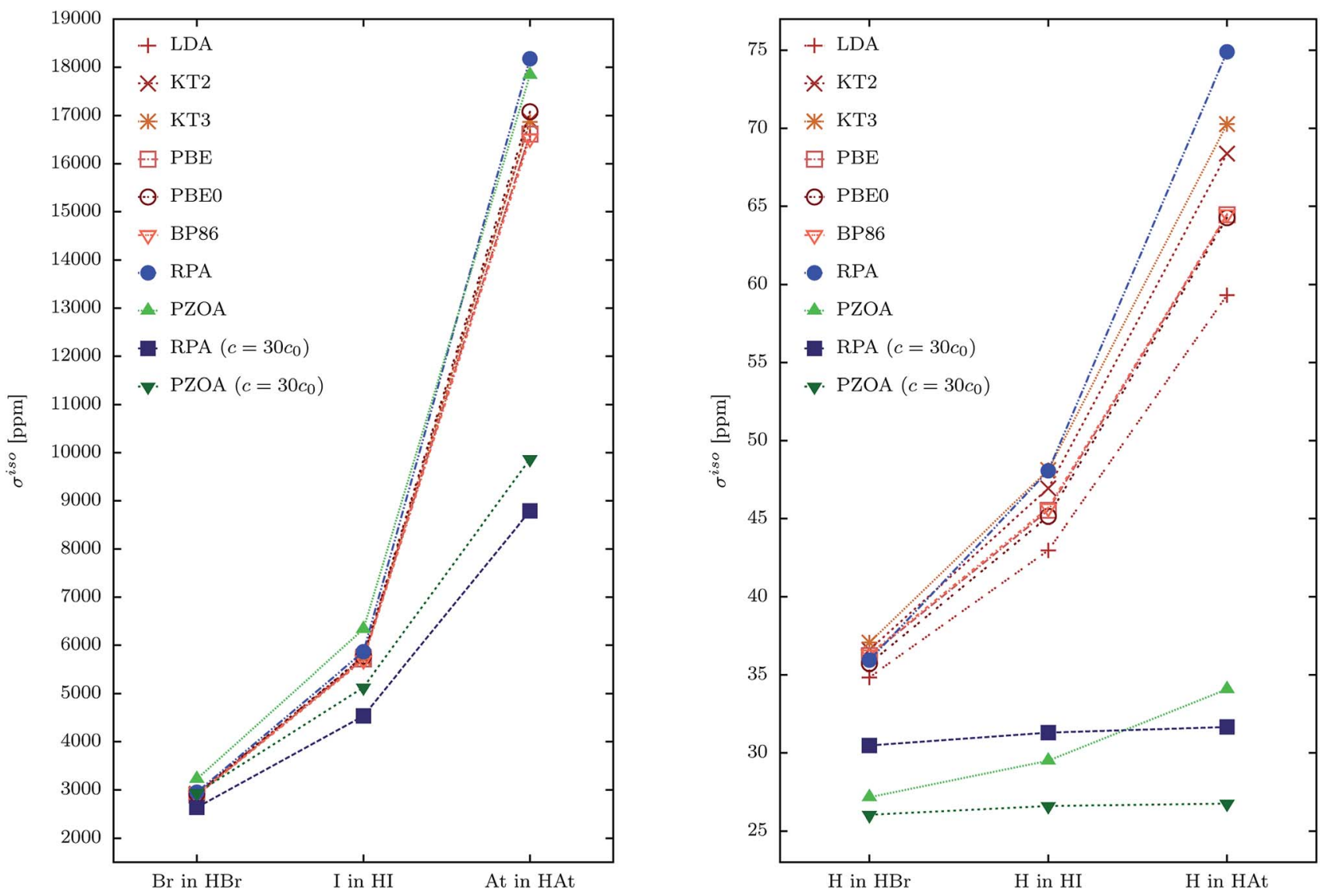

Fig. 6 Relativistic isotropic shielding constants of both nuclei in $\mathrm{HX}$ molecules (X $=\mathrm{Br}$, I, At). Results at PZOA and RPA levels of approach and also employing different DFT functionals are shown. In addition, PZOA and RPA results with $c=30 c_{0}$ are displayed in order to show the behavior of $\boldsymbol{\sigma}^{\text {iso }}$ in the NR limit. All calculations were performed using the GNM, and the results are given in ppm.

compared with those of the formalism of relativistic polarization propagator at the PZOA and RPA levels of approach.

It is worth to mention that the effect of using different optimized geometries for the HAt molecule (see Table 6) is analyzed in Section 4.7.

In Fig. 5 and 6 we observe the size of relativistic and electron correlation effects on SR constants and isotropic shieldings. All values in both figures were obtained employing GNM. It is also observed that, in all cases (but not for $\mathrm{H}$ in $\mathrm{HBr}$ ), the DFT values are always smaller than the RPA ones. In addition, it is clearly shown that the relativistic effects at RPA level (given by the difference between the relativistic RPA values and their equivalent NR RPA which were obtained for $c=30 c_{0}$ ) are positive and directly proportional to $Z_{X}$. This behavior is similar for both properties, $M_{\perp, \mathrm{Y}}$ and $\sigma_{\mathrm{Y}}^{\text {iso }}$.

In the special case of $M_{\perp, \mathrm{At}}$, the relativistic RPA value is $-50.10 \mathrm{kHz}$ (employing GNM), and all DFT values are between $-540 \mathrm{kHz}$ and $-400 \mathrm{kHz}$ (see Table 6). This is in agreement with previous results of Komorovsky and co-authors. ${ }^{53}$ For $\sigma_{\mathrm{At}}^{\text {iso }}$, the RPA calculation (with GNM) gives a result of $18174.03 \mathrm{ppm}$, whereas the DFT values (also with GNM) are between $16525 \mathrm{ppm}$ and $17078 \mathrm{ppm}$. It means that correlation effects are much more important for $M_{\perp, \mathrm{At}}$ than for $\sigma_{\mathrm{At}}^{\text {iso }}$.
The analysis of electron correlation effects within the polarization propagator theory may start considering the zeroth-order or PZOA level of approach and the consistent first order or RPA. ${ }^{37}$ The second-order level of approach or SOPPA is not included here because it is not available in the DIRAC code.

In Fig. 5 we show the behavior of $M_{\perp, \mathrm{X}}$ and $M_{\perp, \mathrm{H}}$ in the whole set of $\mathrm{HX}$ molecules. In the case of $M_{\perp, \mathrm{X}}$ the PZOA approach gives similar values in both regimes, but the relativistic RPA does give a value for $\mathrm{X}=$ At that is far away from its NR counterpart and all DFT functionals. On the other hand, for hydrogen, the pattern of both, the NR PZOA and NR RPA is opposite to that of the relativistic PZOA and RPA. A similar pattern is found for $\sigma_{\mathrm{H}}^{\text {iso }}$ though this time the pattern of the relativistic PZOA and RPA for values of $\sigma_{\mathrm{X}}^{\text {iso }}$ is quite similar to the corresponding DFT values (see Fig. 6). The difference between the patterns of $M_{\perp, \mathrm{x}}$ and $\sigma_{\mathrm{X}}^{\text {iso }}$ for $\mathrm{X}=$ At is due to the contribution of the second term of eqn (15), the atomic contribution, which makes that the RPA value of $\sigma_{\mathrm{At}}^{\text {iso }}$ follows the same tendency as its equivalent DFT values.

On the other hand, the fact that the RPA value of $M_{\mathrm{At}}^{\mathrm{L}(\mathrm{e}-\mathrm{e})}$ is much smaller than its equivalent in DFT makes that the RPA 
value of $M_{\perp, \mathrm{At}}$ is also much smaller, in absolute value, than the DFT ones (see ESI $\dagger$ ). This explains what is shown in Fig. 5.

As can be seen in Table 6 the electron correlation on the NChDE of both, $M_{\perp, A t}$ and $\sigma_{\mathrm{At}}^{\text {iso }}$ follows the same trend from PZOA to RPA as from PZOA to any DFT. The main difference is that the RPA values are little exaggerated. The same behavior is shown in the $\mathrm{ESI}^{\dagger}$ for $\mathrm{HBr}$ and $\mathrm{HI}$.

In the case of the NChDE on $M_{\perp}$ we observe that the RPA value of $\Delta M_{\perp, \mathrm{At}}$ is $-40.15 \mathrm{kHz}$, and DFT results are between -12 $\mathrm{kHz}$ and $-21 \mathrm{kHz}$, depending on the employed functional. These results indicate that a proper analysis of the $\mathrm{NChDE}$ of the $M_{\perp, \mathrm{At}}$ in heavy-element-containing molecules must include correlation effects. $\Delta M_{\perp, \mathrm{At}}$ is of the order of $5 \%$ for the PBE0 level of theory.

On the other hand, the RPA value of $\Delta \sigma_{\mathrm{At}}^{\text {iso }}$ is $-645 \mathrm{ppm}$, but its values for different functionals are between $-597 \mathrm{ppm}$ and $-564 \mathrm{ppm}$. These results indicate that the NChDE in $\sigma_{\mathrm{At}}^{\text {iso }}$ are, in relative terms, less influenced by correlation effects than the NChDE in $M_{\perp, \mathrm{At}}$. This behavior is explained by the fact that the NChDE on shieldings is mostly given by $\Delta \sigma_{\mathrm{Y}}^{\text {atom }}$ (as it was shown in Section 4.3) and the fact that the shielding of free atoms is almost not influenced by correlation effects. In this case $\Delta \sigma_{\mathrm{At}}^{\text {iso }}$ at PBE0 level is about $3 \%$.

The correlation effect that influence the total NChDE does reduce it in both properties. For At, employing the PBE0 functional, $\Delta \sigma^{\text {iso }}$ is reduced in $8.12 \%$ but in $\Delta M_{\perp}$ its reduction is of
$49.01 \%$. In other words, both, the NChDE and the correlation effects are not independent each other, as one may expect from our finding that the NChDE is almost purely relativistic, together with some previously published results where it was shown that correlation effects are not independent of relativistic effects. ${ }^{22}$

In the ESI $\dagger$ we give a table similar to Table 6, where results of calculations of $M_{\perp}$ and $\sigma^{\text {iso }}$ for the HBr and HI molecules at RPA and DFT levels of approach are given. In that cases, a similar behavior is found though much reduced in both, absolute and percentage values.

\subsection{Contributions of (SS $\mid \mathrm{SS})$ integrals and Gaunt interactions}

Given that the NChDE are relatively small for $M_{\perp, \mathrm{At}}$ and $\sigma_{\mathrm{At}}^{\text {iso }}$, we want to go one step further and analyze how large are the contributions of the two-electron (SS|SS) integrals and the electron-electron Gaunt interactions in both properties.

For $M_{\perp, \mathrm{At}}$ and using GNM we observe in Table 6 that the largest contribution appears at RPA level of approach, which is of the order of $7 \%$. At PBE0 its contribution is less than $0.1 \%$. Furthermore the addition of both contributions, i.e. the (SS|SS) integrals and the Gaunt interactions, to the NChDE gives a total variation of the order of $1.5 \%$ at both levels of theory, RPA and PBE0.

Table 7 Dirac-Coulomb-Kohn-Sham-DFT calculations of nuclear spin-rotation constants and isotropic shieldings of At and $\mathrm{H}$ in the HAt molecule, employing two different internuclear distances

\begin{tabular}{|c|c|c|c|c|c|c|c|}
\hline \multicolumn{8}{|c|}{$M_{\perp, \mathbf{A t}}[\mathrm{kHz}]$} \\
\hline PNM & 1.7209 & -496.6202 & -459.2587 & -464.0807 & -486.2404 & -378.4707 & -504.2249 \\
\hline NChDE & & -12.1990 & -16.1038 & -18.4934 & -15.3513 & -20.2567 & -14.8161 \\
\hline PNM & 1.7486 & -515.1978 & -473.6118 & -477.4298 & -503.0270 & -387.7653 & -522.0403 \\
\hline GNM & & -527.7124 & -490.3322 & -496.7065 & -518.9058 & -408.9899 & -537.3335 \\
\hline \multicolumn{8}{|c|}{$M_{\perp, \mathbf{H}}[\mathbf{k H z}]$} \\
\hline PNM & 1.7209 & 86.7008 & 108.5049 & 113.8844 & 97.3378 & 100.0081 & 97.2126 \\
\hline GNM & & 86.6470 & 108.4310 & 113.8095 & 97.2796 & 99.9353 & 97.1555 \\
\hline NChDE & & -0.0538 & -0.0739 & -0.0749 & -0.0582 & -0.0727 & -0.0571 \\
\hline PNM & 1.7486 & 87.8273 & 111.1163 & 116.8943 & 98.9913 & 102.3894 & 98.7845 \\
\hline GNM & & 87.7737 & 111.0420 & 116.8193 & 98.9336 & 102.3158 & 98.7279 \\
\hline GNM & & 16712.2401 & 16907.5696 & 16868.1533 & 16758.3969 & 17105.0952 & 16683.6181 \\
\hline NChDE & & -563.5204 & -591.6753 & -596.9808 & -576.5314 & -591.6493 & -574.6446 \\
\hline PNM & 1.7486 & 17121.2399 & 17360.2991 & 17328.5135 & 17185.8907 & 17581.0419 & 17102.6616 \\
\hline GNM & & 16556.2251 & 16765.9612 & 16727.985 & 16606.8817 & 16985.2613 & 16525.8056 \\
\hline NChDE & & -565.0148 & -594.3379 & -600.5285 & -579.009 & -595.7806 & -576.856 \\
\hline \multicolumn{8}{|c|}{$\sigma_{\mathbf{H}}^{\text {iso }}[\mathrm{ppm}]$} \\
\hline PNM & 1.7209 & 58.6675 & 68.244 & 70.3058 & 63.3212 & 63.9706 & 63.3368 \\
\hline GNMss & & 58.6459 & 68.2163 & 70.2789 & 63.2993 & 63.9433 & 63.3155 \\
\hline NChDE & & -0.0216 & -0.0277 & -0.0269 & -0.0219 & -0.0273 & -0.0213 \\
\hline PNM & 1.7486 & 59.6896 & 69.9431 & 72.1216 & 64.5404 & 65.4644 & 64.5248 \\
\hline GNM & & 59.668 & 69.9155 & 72.0952 & 64.5189 & 65.437 & 64.504 \\
\hline
\end{tabular}


In the case of the isotropic shielding of At, the contribution of both (SS|SS) integrals and Gaunt interactions at both, RPA and PBE0 levels of theory, is close to $-0.15 \%$. In this case its contribution to the NChDE is $\simeq 0.3 \%$ at $\mathrm{RPA}$ and $\simeq 0.37 \%$ at PBE0.

We should stress here that the contribution of Gaunt interactions are a little bit larger than the contributions of (SS|SS) integrals.

\subsection{Internuclear distance}

As it was expressed in Sections 3 and 4.5, all the calculations that involve the HAt molecule were performed employing different optimized geometries according to different levels of theory (RPA or the chosen DFT functionals). An analysis of the effects of the variation of distances in the calculations of shieldings and SR for the HAt molecule must therefore be given.

In Table 7 we display the values of SR and isotropic shieldings of $\mathrm{H}$ and At, employing the smallest and largest internuclear distances optimized within the DFT level of approach: $1.7209 \AA$ (KT3) and $1.7486 \AA$ (BP86) (see Table 6).

As can be seen, the influence of the variation of $d(\mathrm{H}-\mathrm{At})$ on the NChDE of both properties and also on the properties themselves is very small. It means that the main results of Section 4.5 are still valid considering this effect.

Whereas it should be interesting to analyze in more detail the importance of the effect of varying the internuclear distance on SR and shieldings (it could give insights about vibrational effects) it is out of the scope of the present work.

\section{Concluding remarks}

This work was focused on getting new insights about the electronic origin and the size of the NChDE on spin-rotation constants (for the first time), and also on nuclear magnetic shieldings. We also investigated the effect of electron correlation and electron-electron Gaunt interactions on both response properties, and whether such effects are related each other.

We have applied several recent theoretical tools that make easier the analysis. One of them is the possibility to separate the four-component expression of response properties, within the relativistic polarization propagator formalism, into two terms: the (e-e), which is paramagnetic-like due to it goes exactly to the paramagnetic contribution when $c \rightarrow \infty$, and the (p-p), which is diamagnetic-like because it goes to the diamagnetic contribution when $c \rightarrow \infty$. We also applied the recent generalization of the Flygare's relationship to the relativistic framework. This relationship is such that the nuclear magnetic shielding of a nucleus in a given molecular system can be expressed as the sum of three terms: its spin-rotation constant, the shielding of the nucleus in the free atom, and the last term that have two new contributions: one that can be related, for linear molecules, with the electronic spin part of the spin-rotation constant, and the second one which is a response property for the nucleus in the free atom.

Our main results are the following:
(1) The (e-e) terms of both properties are more dependent of the NChDE than the (p-p) ones.

(2) The NChDE on both properties are almost completely relativistic in its origin.

(3) Given that, in the NR limit only $M_{\perp, \mathrm{Y}}^{\mathrm{L}(\mathrm{e}-\mathrm{e})}$ and $\sigma_{\mathrm{Y}}^{\text {atom(p-p) }}$ are nonzero, $\Delta M_{\perp, \mathrm{Y}}^{\mathrm{L}(\mathrm{e}-\mathrm{e})}$ and $\Delta \sigma_{\mathrm{Y}}^{\text {atom }(\mathrm{p}-\mathrm{p})}$ are very small and $\Delta \sigma_{\perp, \mathrm{Y}}^{(\mathrm{e}-\mathrm{e})}=\frac{m_{\mathrm{p}} I}{g_{\mathrm{Y}}} \Delta M_{\perp, \mathrm{Y}}^{\mathrm{L}(\mathrm{e}-\mathrm{e})}$.

(4) The following are the terms which mainly contribute to the relativistic effects on

(a) Shielding $\left|\Delta^{\mathrm{rel}} \sigma_{\mathrm{Y}^{-}}^{\text {atom }(\mathrm{e}-\mathrm{e})}\right|>\frac{m_{\mathrm{p}} I}{g_{\mathrm{Y}}}\left|\Delta^{\mathrm{rel}} M_{\perp, \mathrm{Y}}^{\mathrm{S}(\mathrm{e}-\mathrm{e})}\right|>\frac{m_{\mathrm{p}} I}{g_{\mathrm{Y}}}\left|\Delta^{\mathrm{rel}} M_{\perp, \mathrm{Y}}^{\mathrm{L}(\mathrm{e}-\mathrm{e})}\right|>\ldots$

(b) Spin-rotation constants: $\left|\Delta^{\mathrm{rel}} M_{\perp, \mathrm{Y}}^{\mathrm{S}(\mathrm{e}-\mathrm{e})}\right|>\left|\Delta^{\mathrm{rel}} M_{\perp, \mathrm{Y}}^{\mathrm{L}(\mathrm{e}-\mathrm{e})}\right|$.

In the same manner, the following are the main contributions to the NChDE on

(a) Shielding constants: $\left|\Delta \sigma_{\mathrm{Y}^{-}}^{\text {atom }(\mathrm{e}-\mathrm{e})}\right|>\frac{m_{\mathrm{p}} I}{g_{\mathrm{Y}}}\left|\Delta M_{\perp, \mathrm{Y}}^{\mathrm{S}(\mathrm{e}-\mathrm{e})}\right|>\frac{m_{\mathrm{p}} I}{g_{\mathrm{Y}}}\left|\Delta M_{\perp, \mathrm{Y}}^{\mathrm{L}(\mathrm{e}-\mathrm{e})}\right|>\ldots$

(b) Spin-rotation constants: $\left|\Delta M_{\perp, \mathrm{Y}}^{\mathrm{S}(\mathrm{e}-\mathrm{e})}\right|>\left|\Delta M_{\perp, \mathrm{Y}}^{\mathrm{L}(\mathrm{e}-\mathrm{e})}\right|$.

(5) Results of calculations performed at relativistic DFT/PBE0 level of theory are the closest to the experimental values of spinrotation constants of $\mathrm{Br}$ and $\mathrm{I}$. In the case of $\mathrm{H}$ the best reproduction of experimental values is given by the DFT/LDA functional.

(6) Electron correlation effects are very important for spinrotation tensors. Values at DFT/PBE0 level of theory are eight times the values at relativistic RPA level of theory for At in HAt.

(7) When both effects, electron correlation and the NChDE are included altogether, the NChDE on $M_{\perp, \text { At }}$ is $5 \%$ of its total correlated value at relativistic DFT/PBE0 level of theory. In the case of relativistic RPA calculations, the NChDE is $80 \%$.

(8) From the fact that the NChDE mostly is a relativistic effect, we can expect, and actually observe, that this effect is not independent of the electron correlation.

(9) The introduction of (SS|SS) type integrals and electronelectron Gaunt interaction modifies a few percent of the NChDE of both properties, and less than $0.3 \%$ of $\sigma_{\mathrm{At}}^{\text {iso }}$.

\section{Conflicts of interest}

There are no conflicts of interest to declare.

\section{Acknowledgements}

We acknowledge partial support from the Argentinian National Scientific and Technical Research Council (CONICET, Grant PIP 112-20130100361).

\section{References}

1 D. Andrae, in Nuclear Charge Density and Magnetization Distributions, ed. W. Liu, Springer Berlin Heidelberg, Berlin, Heidelberg, 2016, pp. 1-31.

2 L. Visscher and K. G. Dyall, At. Data Nucl. Data Tables, 1997, 67, 207-224. 
3 L. Visscher, T. Saue and J. Oddershede, Chem. Phys. Lett., 1997, 274, 181-188.

4 V. M. Shabaev, J. Phys. B: At., Mol. Opt. Phys., 1993, 26, 11031108.

5 D. Andrae, Phys. Rep., 2000, 336, 413-525.

6 R. Fukuda, M. Hada and H. Nakatsuji, J. Chem. Phys., 2003, 118, 1027-1035.

7 S. Hamaya, H. Maeda, M. Funaki and H. Fukui, J. Chem. Phys., 2008, 129, 224103.

8 E. Malkin, I. Malkin, O. L. Malkina, V. G. Malkin and M. Kaupp, Phys. Chem. Chem. Phys., 2006, 8, 4079-4085.

9 E. Malkin, M. Repiský, S. Komorovský, P. Mach, O. L. Malkina and V. G. Malkin, J. Chem. Phys., 2011, 134, 044111.

10 J. Autschbach, ChemPhysChem, 2009, 10, 2274-2283.

11 S. Moncho and J. Autschbach, J. Chem. Theory Comput., 2010, 6, 223-234.

12 A. F. Maldonado, C. A. Giménez and G. A. Aucar, J. Chem. Phys., 2012, 136, 224110.

13 Y. Kita and M. Tachikawa, Comput. Theor. Chem., 2011, 975, 9-12.

14 V. Arcisauskaite, J. I. Melo, L. Hemmingsen and S. P. A. Sauer, J. Chem. Phys., 2011, 135, 044306.

15 E. Malkin, S. Komorovsky, M. Repisky, T. B. Demissie and K. Ruud, J. Phys. Chem. Lett., 2013, 4, 459-463.

16 I. A. Aucar, S. S. Gomez, J. I. Melo, C. G. Giribet and M. C. Ruiz de Azúa, J. Chem. Phys., 2013, 138, 134107.

17 I. A. Aucar, S. S. Gomez, M. C. Ruiz de Azúa and C. G. Giribet, J. Chem. Phys., 2012, 136, 204119.

18 Y. Xiao and W. Liu, J. Chem. Phys., 2013, 138, 134104.

19 Y. Xiao, Y. Zhang and W. Liu, J. Chem. Theory Comput., 2014, 10, 600-608.

20 I. A. Aucar, S. S. Gomez, C. G. Giribet and G. A. Aucar, Phys. Chem. Chem. Phys., 2016, 18, 23572-23586.

21 I. A. Aucar, S. S. Gomez, C. G. Giribet and G. A. Aucar, J. Phys. Chem. Lett., 2016, 7, 5188-5192.

22 C. A. Giménez, A. F. Maldonado and G. A. Aucar, Theor. Chem. Acc., 2016, 135, 201-212.

23 W. R. Johnson and G. Soff, At. Data Nucl. Data Tables, 1985, 33, 405-446.

24 N. F. Ramsey, Phys. Rev., 1950, 78, 699-703.

25 W. H. Flygare, J. Chem. Phys., 1964, 41, 793-800.

26 W. H. Flygare, Chem. Rev., 1974, 74, 653-687.

27 W. H. Flygare and J. Goodisman, J. Chem. Phys., 1968, 49, 3122-3125.

28 T. D. Gierke and W. H. Flygare, J. Am. Chem. Soc., 1972, 94, 7277-7283.

29 G. A. Aucar and J. Oddershede, Int. J. Quantum Chem., 1993, 47, 425-435.

30 G. A. Aucar, T. Saue, L. Visscher and H. J. A. Jensen, J. Chem. Phys., 1999, 110, 6208-6218.

31 I. A. Aucar, S. S. Gomez, C. G. Giribet and M. C. Ruiz de Azúa, J. Chem. Phys., 2013, 139, 094112.

32 G. A. Aucar, J. I. Melo, I. A. Aucar and A. F. Maldonado, Int. J. Quantum Chem., 2018, 118, e25487.

33 DIRAC, a Relativistic Ab Initio Electronic Structure Program, Release DIRAC16 (2016), written by H. J. Aa. Jensen, R. Bast,
T. Saue, and L. Visscher, with contributions from V. Bakken, K. G. Dyall, S. Dubillard, U. Ekström, E. Eliav, T. Enevoldsen, E. Faßhauer, T. Fleig, O. Fossgaard, A. S. P. Gomes, T. Helgaker, J. Henriksson, M. Iliaš, Ch. R. Jacob, S. Knecht, S. Komorovský, O. Kullie, J. K. Lærdahl, C. V. Larsen, Y. S. Lee, H. S. Nataraj, M. K. Nayak, P. Norman, G. Olejniczak, J. Olsen, Y. C. Park, J. K. Pedersen, M. Pernpointner, R. di Remigio, K. Ruud, P. Sałek, B. Schimmelpfennig, A. Shee, J. Sikkema, A. J. Thorvaldsen, J. Thyssen, J. van Stralen, S. Villaume, O. Visser, T. Winther, and S. Yamamoto (see http://www.diracprogram.org).

34 L. Visscher, Theor. Chem. Acc., 1997, 98, 68-70.

35 N. N. Dutta and S. Majumder, Phys. Rev. A, 2012, 85, 032512. 36 K. Kozioł, C. A. Giménez and G. A. Aucar, J. Chem. Phys., 2018, 148, 044113.

37 G. A. Aucar, R. H. Romero and A. F. Maldonado, Int. Rev. Phys. Chem., 2010, 29, 1-64.

38 P. Raghavan, At. Data Nucl. Data Tables, 1989, 42, 189-291. 39 W. Haynes, CRC Handbook of Chemistry and Physics, CRC Press, 97th edn, 2016.

40 T. H. Dunning, J. Chem. Phys., 1989, 90, 1007-1023.

41 K. G. Dyall, Theor. Chem. Acc., 2006, 115, 441-447.

42 P. Hohenberg and W. Kohn, Phys. Rev. B, 1964, 136, B864B871.

43 S. J. Vosko, L. Wilk and M. Nusair, Can. J. Phys., 1980, 58, 1200-1211.

44 J. P. Perdew, K. Burke and M. Ernzerhof, Phys. Rev. Lett., 1996, 77, 3865-3868.

45 T. W. Keal and D. J. Tozer, J. Chem. Phys., 2003, 119, 30153024 .

46 T. W. Keal and D. J. Tozer, J. Chem. Phys., 2004, 121, 56545660.

47 A. D. Becke, Phys. Rev. A, 1988, 38, 3098-3100.

48 J. P. Perdew and Y. Wang, Phys. Rev. B, 1986, 33, 8800-8802.

49 C. Adamo and V. Barone, J. Chem. Phys., 1999, 110, 61586170.

50 P. J. Mohr, D. B. Newell and B. N. Taylor, Rev. Mod. Phys., 2016, 88, 035009.

$51 \mathrm{~J}$. Autschbach and T. Ziegler, Relativistic Computation of NMR Shieldings and Spin-Spin Coupling Constants, in Advances in NMR, John Wiley and Sons, 2002, vol. 9, pp. 306-323.

52 A. Antušek and M. Jaszuński, Phys. Rev., 2006, 104, 14631474.

53 S. Komorovsky, M. Repisky, E. Malkin, T. B. Demissie and K. Ruud, J. Chem. Theory Comput., 2015, 11, 3729-3739.

54 A. F. Maldonado and G. A. Aucar, J. Phys. Chem. A, 2014, 118, 7863-7875.

55 I. L. Rusakova and L. B. Krivdin, Mendeleev Commun., 2018, 28, 1-13.

56 E. Engel, S. Keller and R. M. Dreizler, Phys. Rev. A, 1996, 53, 1367-1374.

57 R. Schmid, E. Engel, R. Dreizler, P. Blaha and K. Schwarz, Density Functional Theory, Academic Press, 1998, vol. 33, pp. 209-223. 
58 T. Helgaker, M. Jaszuński and M. Pecul, Prog. Nucl. Magn. Reson. Spectrosc., 2008, 53, 249-268.

59 B. Jeziorski, Mol. Phys., 2010, 108, 3043-3054.

60 I. L. Rusakova, Y. Y. Rusakov and L. B. Krivdin, Russ. Chem. Rev., 2016, 85, 356-426.
61 F. P. Van Dijk and A. Dymanus, Chem. Phys. Lett., 1969, 4, 170-172.

62 F. P. Van Dijk and A. Dymanus, Chem. Phys. Lett., 1968, 2, 235-236. 